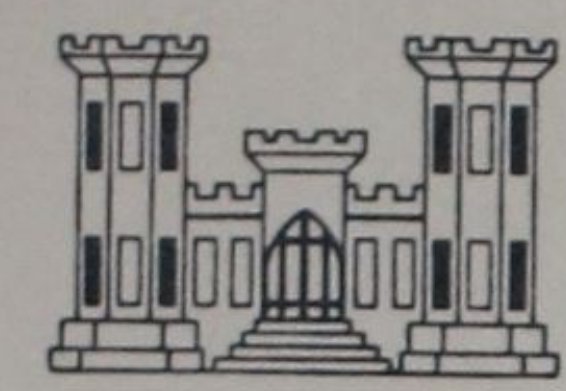

REFERENCE
MISCELLANEOUS PAPER M-76-13

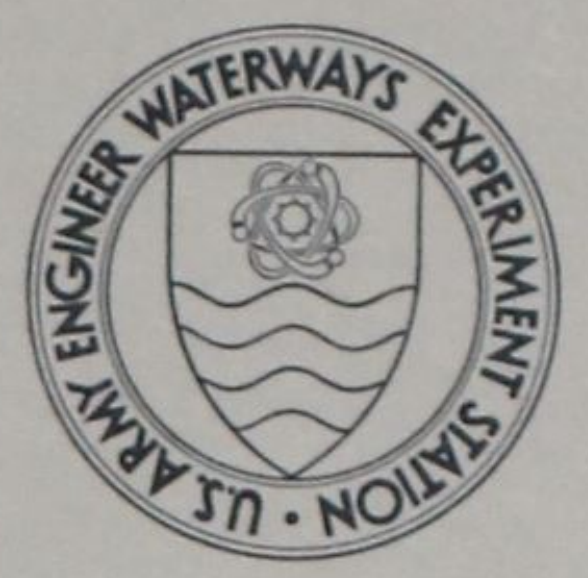

\title{
SEISMIC METHODS OF LOCATING MILITARY GROUND TARGETS
}

by

Daniel H. Cress

Mobility and Environmental Systems Laboratory

U. S. Army Engineer Waterways Experiment Station

P. O. Box 631, Vicksburg, Miss. 39180

June 1976

Final Report

Approved For Public Release; Distribution Unlimited
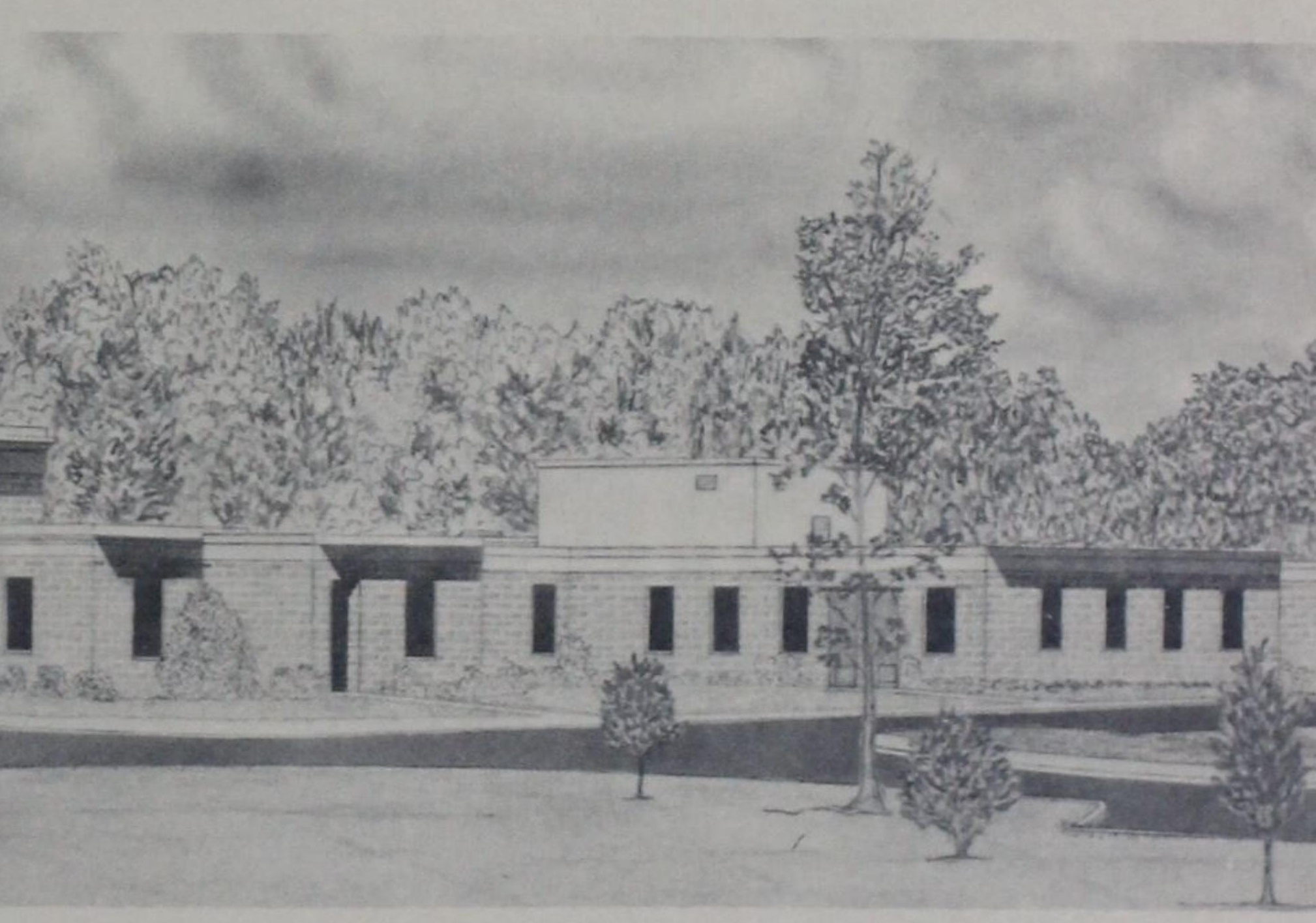

Sponsored by U. S. Army Materiel Development and Readiness Command

Alexandria, Virginia 22333

Under Project ITI62II2A528, Task 02

TECHNICAL MRY BRANCH

US ARMY ENGINEER INFORMATION CENTER 
Unclassified

SECURITY CLASSIFICATION OF THIS PAGE (When Data Entered)

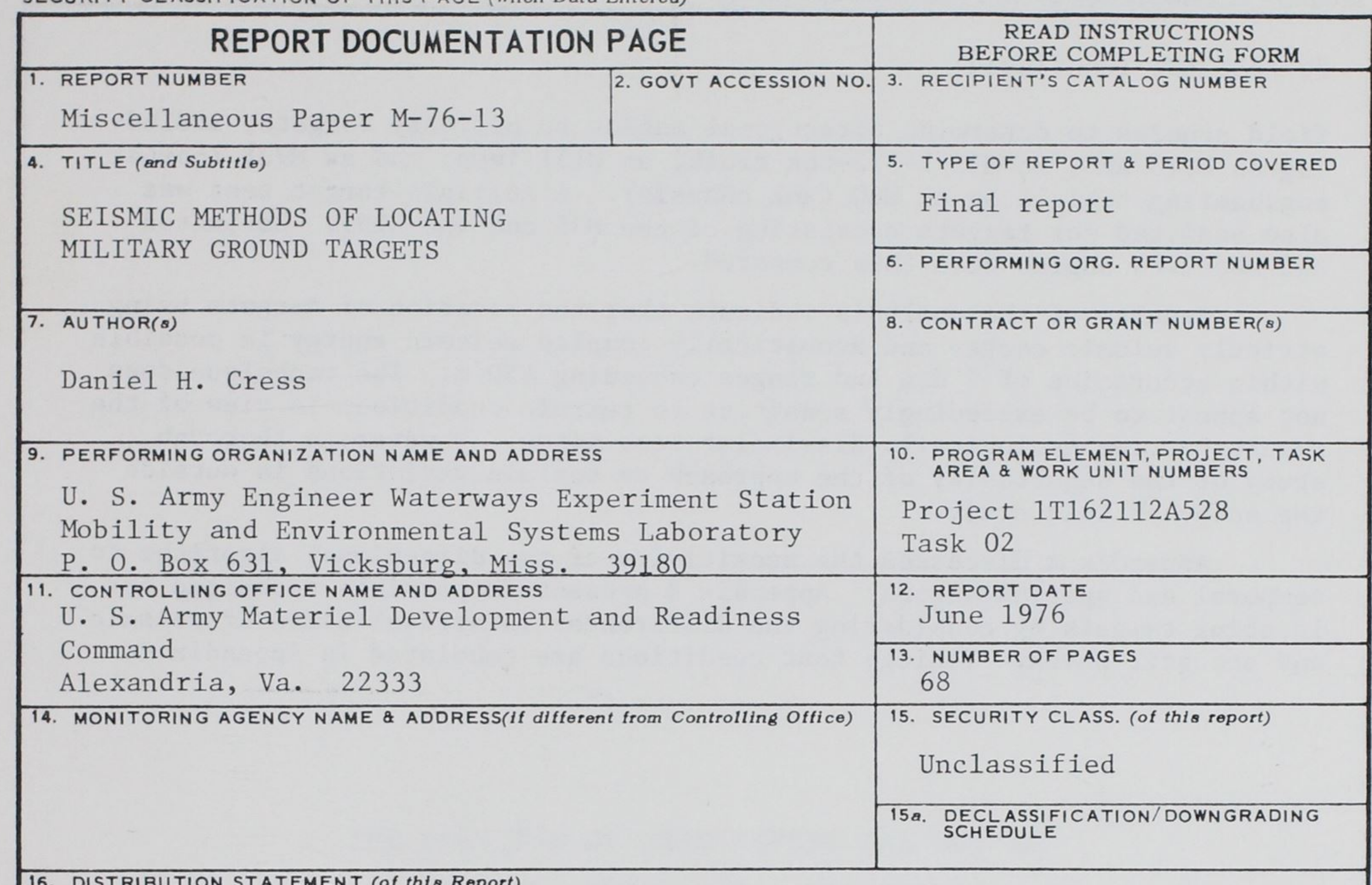

16. DISTRIBUTION STATEMENT (of this Report)

Approved for public release; distribution unlimited.

17. DISTRIBUTION STATEMENT (of the abstract ontered in Block 20, if different from Report)

18. SUPPLEMENTARY NOTES

19. KEY WORDS (Continue on reverse side if necessary and identify by block number)

Geophones

Ground targets

Military operations

Seismic waves

20. ABSTRACT (Continue an reverse side if necossary and tdentify by block number)

An approach for locating military ground targets with a triangular array of geophones is presented. An algorithm that relates the characteristics of the signature received at each geophone to the direction of the target is derived. The signatures are then processed by two methods, which have differing degrees of complexity, to estimate target position.

The target-location system (including array deployment) was used in

(Continued) 


\section{ABSTRACT (Continued)}

field studies to determine directional angles to military targets, including an M113 APC, an M35 2-1/2-ton truck, an M151 jeep, and an M728 (combat engineering vehicle on an M60 tank chassis). A multiple-target test was also analyzed for targets consisting of the M35 and the M151. Estimated and measured angles were then compared.

Results of the analysis indicate that the location of targets using strictly seismic energy and acoustically coupled seismic energy is possible within accuracies of $5 \mathrm{deg}$ and ranges exceeding $450 \mathrm{~m}$. The technique does not appear to be exceedingly sensitive to terrain conditions in view of the results at two geologically dissimilar test sites. However, a thorough study of the sensitivity of the approach to terrain variations is outside the scope of this study.

Appendix A discusses the sensitivity of the directional algorithm to temporal and spatial errors. Appendix B presents a possible technique for locating targets by considering the differences in arrival times of seismic and acoustic waves. Vehicle test conditions are tabulated in Appendix C. 
THE CONTENTS OF THIS REPORT ARE NOT TO BE USED FOR ADVERTISING, PUBLICATION, OR PROMOTIONAL PURPOSES. CITATION OF TRADE NAMES DOES NOT CONSTITUTE AN OFFICIAL ENDORSEMENT OR APPROVAL OF THE USE OF SUCH COMMERCIAL PRODUCTS. 


\section{PREFACE}

Personnel of the Environmental Systems Division (ESD), Mobility and Environmental Systems Laboratory (MESL), U. S. Army Engineer Waterways Experiment Station (WES), conducted this study for the U. S. Army Materiel Development and Readiness Command in furtherance of DA Project No. 1T162112A528, "Environmental Effects on Materie1," Task 02, "Terrestrial Effects on Materiel Development," and in support of the Project Manager, Remotely Monitored Battlefield Sensor Systems, under Support Agreement No. AMC-PM-RBS-74-06-18.

The study was conducted during the period August 1973 - September 1974 under the general supervision of Messrs. W. G. Shockley, Chief, MESL, and B. O. Benn, former Chief, Environmental Research Branch (ERB) and now Chief, ESD, and under the direct supervision of Mr. J. R. Lundien, former Chief, ERB, and now Senior Electrical Engineer, ESD. Project leader was Dr. D. H. Cress. The computer programs used in the report were compiled by Messrs. J. R. Stabler and E. A. Baylot, ERB. The report was prepared by Dr. Cress. Discussions with Messrs. Carl Bennett and Raymond Fidler of the Naval Coastal Systems Laboratory, Panama City, Florida, concerning analogous location techniques using pressure waves in water were very helpful.

Director of WES during this study and preparation of the report was COL G. H. Hilt, CE. Technical Director was Mr. F. R. Brown. 
$\underline{\text { Page }}$

PREFACE. • • • • • • • • • . • • • . . . . . . . . 2

CONVERSION FACTORS, METRIC (SI) TO U. S. CUSTOMARY AND

U. S. CUSTOMARY TO METRIC (SI) UNITS OF MEASUREMENT. • • • . . 4

PART I: INTRODUCTION. . . . . . . . . . . . . . 5

Background ..................... 5

Approach and Scope of This Study . . . . . . . . 6

PART II: CONCEPTS FOR TARGET LOCATION . . . . . . . . . . 10

Derivation of Directional Algorithm. . . . . . . . 12

Methods of Signature Analysis. . . . . . . . . . 18

Sources of Error in Calculating Target Direction . . . . 23

PART III: APPLICATION OF SIGNAL-PROCESSING TECHNIQUES TO

SIGNATURES OF MOVING TARGETS. • . . . . . . . 25

Data Collection. . . . . . . . . . . . . 25

Results of Signature Analysis. . . . . . . . . . 30

PART IV: CONCLUSIONS AND RECOMMENDATIONS. . . . . . . . . . 44

Conclusions... . . . . . . . . . . . . 44

Recommendations. . . . . . . . . . . . . . 44

TABLES $1-7$

APPENDIX A: SENSITIVITY OF THE DIRECTIONAL ALGORITHM

TO TEMPORAL AND SPATIAL ERRORS. • . . . . . . . A1

Instrumentation and Signal-Processing Errors . . . . . . A1

Errors Due to Plane Wave Assumption. . . . . . . . . A4

APPENDIX B: TARGET LOCATION USING THE DIFFERENCES IN ARRIVAL

TIMES OF SEISMIC AND ACOUSTIC WAVES . . . . . . . B1

APPENDIX C: TABULATION OF SINGLE- AND MULTIPLE-TARGET

TEST CONDITIONS . . . . . . . . . . . C1

TABLE C1 
CONVERSION FACTORS, METRIC (SI) TO U. S. CUSTOMARY AND

U. S. CUSTOMARY TO METRIC (SI) UNITS OF MEASUREMENT

Units of measurement used in this report can be converted as follows:

Multiply

By

To Obtain

Metric (SI) to U. S. Customary

millimetres

$0.03937007 \quad$ inches

centimetres

$0.03937007 \quad$ inches

metres

3.280839

feet

kilometres

0.6213711

miles (U. S. statute)

metres per second

3.280839

feet per second

kilometres per hour

0.6213711

miles (U. S. statute) per hour

U. S. Customary to Metric (SI)

tons (short)

907.1847

kilograms

inches per second

25.4

millimetres per second

degrees (angular)

0.01745329

radians 


\section{SEISMIC METHODS OF LOCATING MILITARY GROUND TARGETS}

\section{PART I: INTRODUCTION}

\section{$\underline{\text { Background }}$}

1. In its effort to acquire the capability to detect and locate ground-based targets, the Army has developed two concepts, one in which an array of unattended seismic sensors is deployed in a region of surveillance to detect and locate continuously moving targets (men, vehicles, etc.) and the other in which acoustic sensors are placed around the region of surveillance to locate firing artillery. Moving targets are located by monitoring the activations of the individual sensors in the array, the accuracy depending upon the range at which the sensors are activated. The individual sensors are spaced such that the activations allow quasi-continuous tracking of the target without an overlapping of the ranges of activation (truly continuous tracking by this method is not possible). Thus, a large number of sensors are necessary. Targets generating impulsive signatures, such as firing artillery, can be located using three or more sensors. The time of arrival of the acoustic signature at each sensor is noted, and the artillery is located by uniquely relating the differences in these arrival times to the coordinates of the target. The difference in arrival times between any two sensors can be used to construct hyperbolic lines of possible target positions. Intersection points for the constructed lines of several pairs of sensors mark the most likely position of the target. A problem arises if the ranges from multiple firing locations to the sensors are such that the ordering of the arrival of the acoustic signature at a given sensor is different from the ordering of the arrivals at other sensors. The sensitivity of the instrumentation to the intensity of the signals and the sensitivity of the algorithms to errors in the arrival times are such that the region of surveillance must be surrounded by sensors. Thus, current target-location systems require that the 
target be within the area bounded by the arrays of sensors (seismic or acoustic) and that the region of surveillance be accessible to personnel installing them.

\section{Approach and Scope of This Study}

2. In a search for improved methods of using seismic energy to locate ground targets, this study has been directed toward evaluating the feasibility of using a target-location system consisting of a group of geophones, i.e. three geophones configured such that a geophone is placed at each of the vertices of an equilateral triangle whose sides are oriented to a specified reference. The geophones are spaced less than $7 \mathrm{~m}$ apart; this array will be referred to herein as a short-based array. A target can be located in a two-dimensional space, as shown in Figure 1, provided the range from some reference point (sensor location) to the target and the directional angle (i.e. the angle between the line from the sensor location to the target and some reference direction) are known. There are several concepts that may be used for determining target location, as will be discussed later. However, the directional angle from one or more short-based arrays must always be obtained. By determining the phase shift in the seismic wave train received at each geophone, the direction to the target can be calculated. Since seismic waves in the ground and acoustic energy that may couple with the ground at the geophone travel at much higher velocities than do most land vehicles (targets), the travel distances for moving targets will be small during the time the signal travels from the target to the geophone. Therefore, if processing times are kept short, the same techniques used for a stationary target can be used for a moving target.

3. The use of short-based arrays of geophones for determining direction from an array to a target has not previously been rigorously investigated; however, the concept has been used successfully for tracking targets that generate pressure waves in water. Furthermore, 


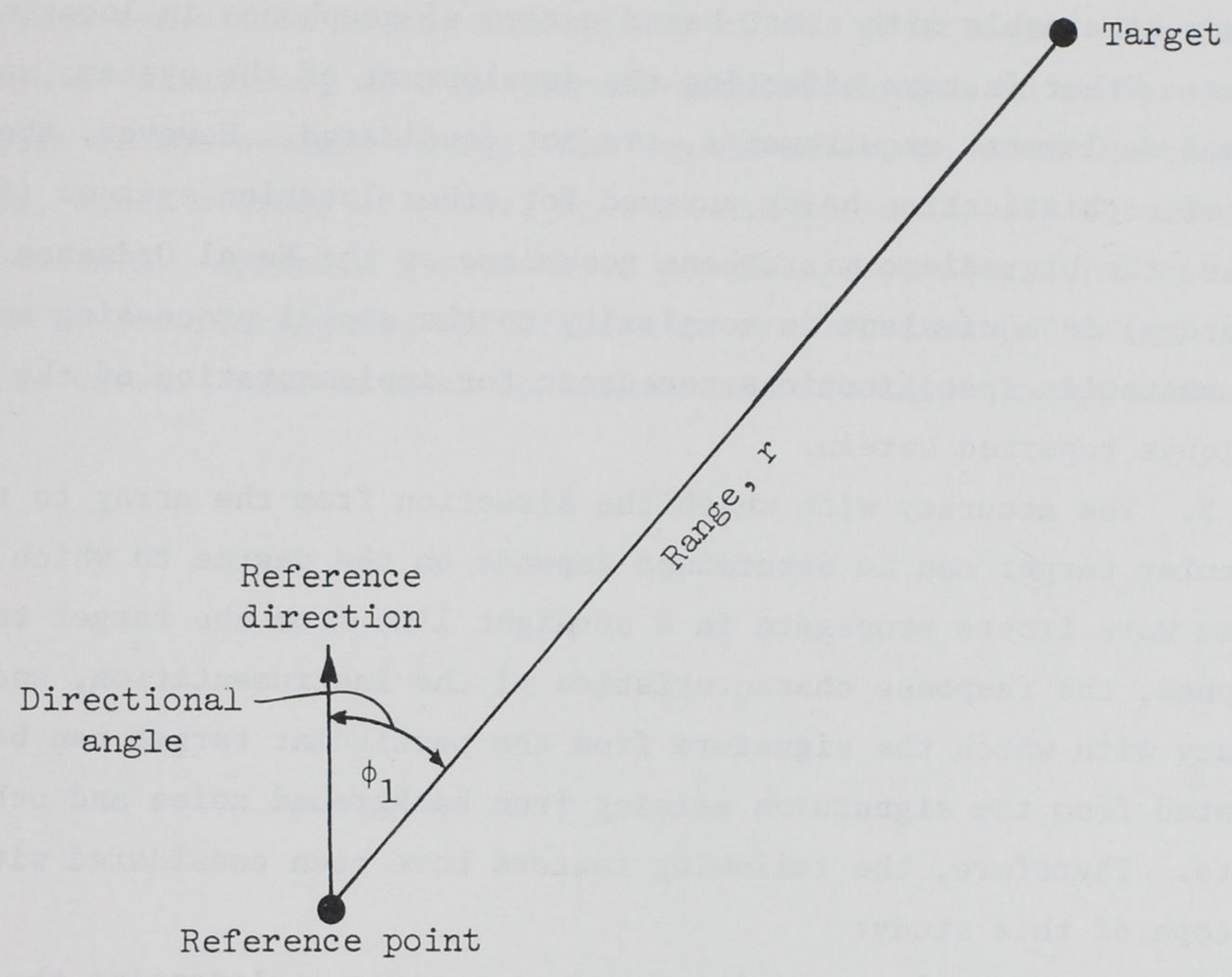

Figure 1. Parameters (i.e. directional angle and range) required for location of a target in a two-dimensional space 
the Naval Coastal Systems Laboratory* has demonstrated that an array of hydrophones can be used to track several different energy sources simultaneously, thus offering the hope that the approach can be used to locate multiple targets using signatures in seismic media as well.

4. The measure of feasibility, as treated in this report, is the accuracy attainable with short-based arrays of geophones in locating targets. Other factors affecting the development of the system, such as cost and deployment requirements, are not considered. However, the level of sophistication being pursued for other location systems (for example, the bigradient microphone technique at the Naval Ordnance Laboratory) is equivalent in complexity to the signal processing and instrumentation specifications necessary for implementation of the techniques reported herein.

5. The accuracy with which the direction from the array to the particular target can be determined depends on the degree to which the seismic wave fronts propagate in a straight line from the target to the geophones, the response characteristics of the instrumentation, and the accuracy with which the signature from the particular target can be separated from the signatures arising from background noise and other targets. Therefore, the following factors have been considered within the scope of this study:

a. Signal-processing requirements for implementing the target-location technique in single- and multiple-target environments.

b. The relation between design parameters for the shortbased array (i.e. spacing between geophones, spatial and phase reponse of geophones, and phase response of amplifiers) and accuracy.

6. To address the factors listed above, two concepts of target location are presented in Part II, both of which require determination of the directional angle. A directional algorithm is derived for the short-based array. Two techniques of signal processing for application

* C. M. Bennett, "The Directional Analysis of Ocean Waves," Informal Report NCSL 144-72, Dec 1972, Coastal Systems Laboratory, Panama City, F1a. 
of the directional algorithm are presented; they differ in their degrees of complexity. In Part III these techniques are applied to test data obtained with wheeled and tracked vehicles in two test programs at two geologically dissimilar sites (Vicksburg, Mississippi, and Fort Carson, Colorado). Conclusions and recommendations are presented in Part IV. A thorough evaluation of the sensitivity of the directional algorithm to terrain variations, such as terrain-induced changes in the propagation path of the seismic energy, was beyond the scope of this study. However, other sources of error, spatial and temporal, are discussed in Appendix A. Appendix B presents a possible technique for locating targets by considering the differences in arrival times of seismic and acoustic waves. Vehicle test conditions are tabulated in Appendix C. 


\section{PART II: CONCEPTS FOR TARGET LOCATION}

7. A target can be located by obtaining the range and directional angle relative to a reference point and reference direction (Figure 1). The reference point can be defined as the location of a short-based array of seismic sensors. The target can then be located by several schemes. Among the concepts of location are:

a. Location by triangulation in which the directional angles to a particular target from two spatially separated short-based arrays are determined (Figure 2). The point of intersection of the two lines defined by the directional angles is the estimated location of the target. Triangulation uniquely determines the two coordinates required for location because the directional angles are determined from the data received at the reference array, and the range is uniquely defined by the distance from the reference array to the intersection of the lines along the directional angles determined by the spatially separated arrays.

b. Ranging from a short-based array to a target by monitoring the difference in time of arrival of two modes of energy transport (such as seismic and acoustic energies) having differences in their respective velocities of propagation. This technique of target location appears applicable only to impulsive targets, such as firing artillery and impacts. It is discussed further in Appendix B.

It should be noted that the results of the study reported herein are more applicable to target location by triangulation than to target location by ranging, since the data analyzed in this report consist of signatures of moving targets rather than impulsive (short-lifetime) signatures. However, the ranging technique is included in Appendix B because it appears to be a possible approach to location of impulsive sources based upon a preliminary examination of seismic and acoustic data.

8. Both of the approaches to target location described in the previous paragraph require that the directional angles relative to two spatially separated short-based arrays (paragraph 7a) or one short-based array (paragraph 7b) be determined. The feasibility of using short-based arrays to determine the location of a target can, to a certain degree, be reduced to evaluating the accuracy with which a short-based array can be 


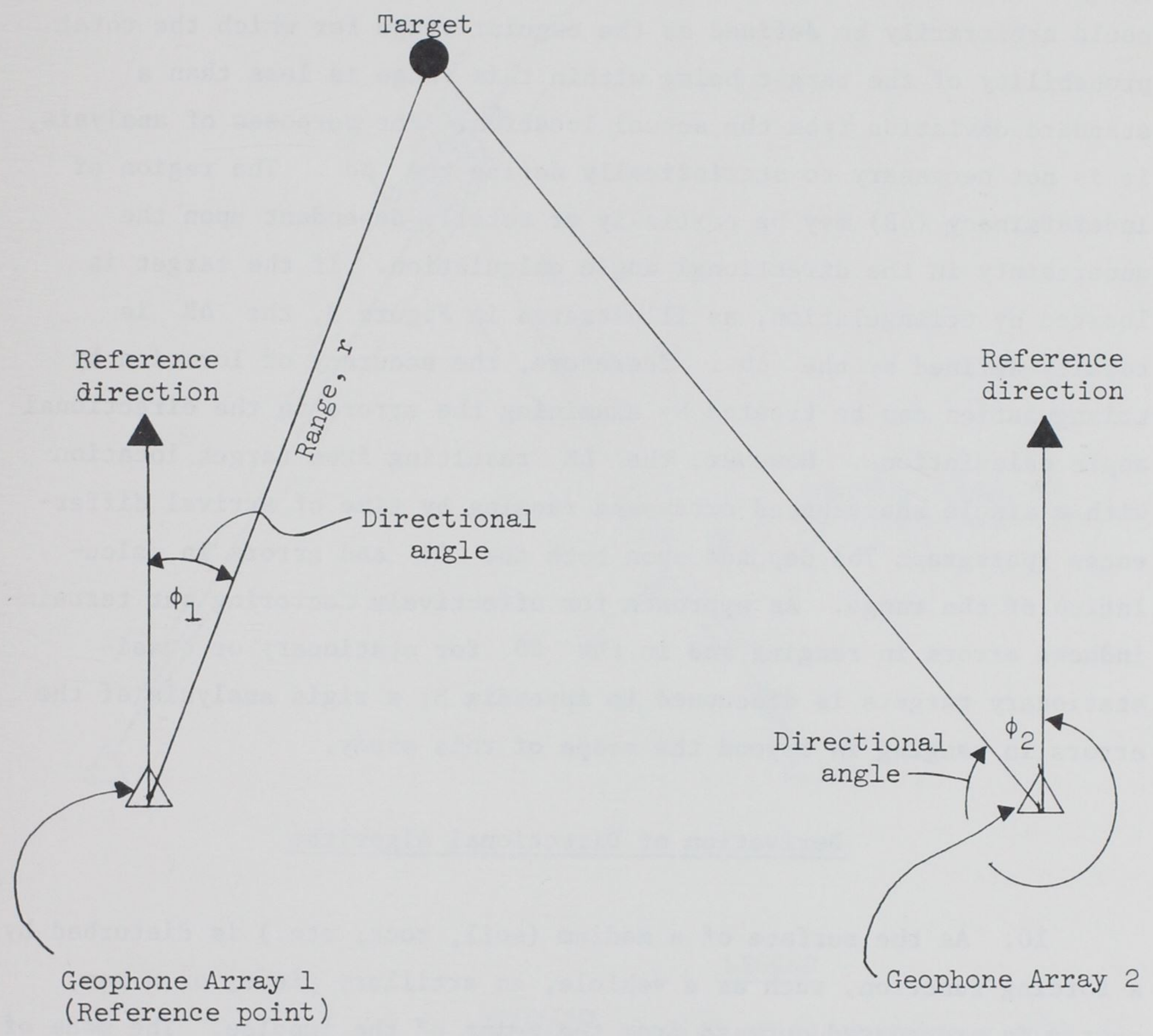

Figure 2. Concept of target location by triangulation 
used to determine the directional angle to the target. Therefore, the primary effort of this study was such an evaluation.

9. The accuracy with which a short-based array can be used to obtain the directional angle can be described by some angular range of indeterminacy $(\Delta \theta)$ (Figure 3). The $\Delta \theta$ is probabilistic in nature and could arbitrarily be defined as the angular range for which the total probability of the target being within this range is less than a standard deviation from the actual location. For purposes of analysis, it is not necessary to statistically define the $\Delta \theta$. The region of indeterminacy $(\Delta R)$ may be partially or totally dependent upon the uncertainty in the directional angle calculation. If the target is located by triangulation, as illustrated in Figure 3 , the $\Delta \mathrm{R}$ is totally defined by the $\Delta \theta$. Therefore, the accuracy of location by triangulation can be treated by examining the errors in the directional angle calculations. However, the $\Delta \mathrm{R}$ resulting from target location with a single short-based array and ranging by time of arrival differences (paragraph 7b) depends upon both the $\Delta \theta$ and errors in calculation of the range. An approach for effectively factoring out terraininduced errors in ranging and in the $\Delta \theta$ for stationary or quasistationary targets is discussed in Appendix B; a rigid analysis of the errors in ranging is beyond the scope of this study.

\section{Derivation of Directional Algorithm}

10. As the surface of a medium (soil, rock, etc.) is disturbed by a forcing function, such as a vehicle, an artillery piece, or a man, energy is propagated outward from the point of the impulse. The mode of propagation can be Rayleigh (i.e. surface) waves, transverse waves, or compressional waves. The extent to which cylindrical symmetry (i.e. radial symmetry about an axis normal to the surface through the point of the impulse) is retained by the wave front depends upon seismic uniformity of the propagating medium. The medium is seismically uniform if the velocity of propagation is not spatially dependent. The amount of displacement of the medium from equilibrium is a function of the range 


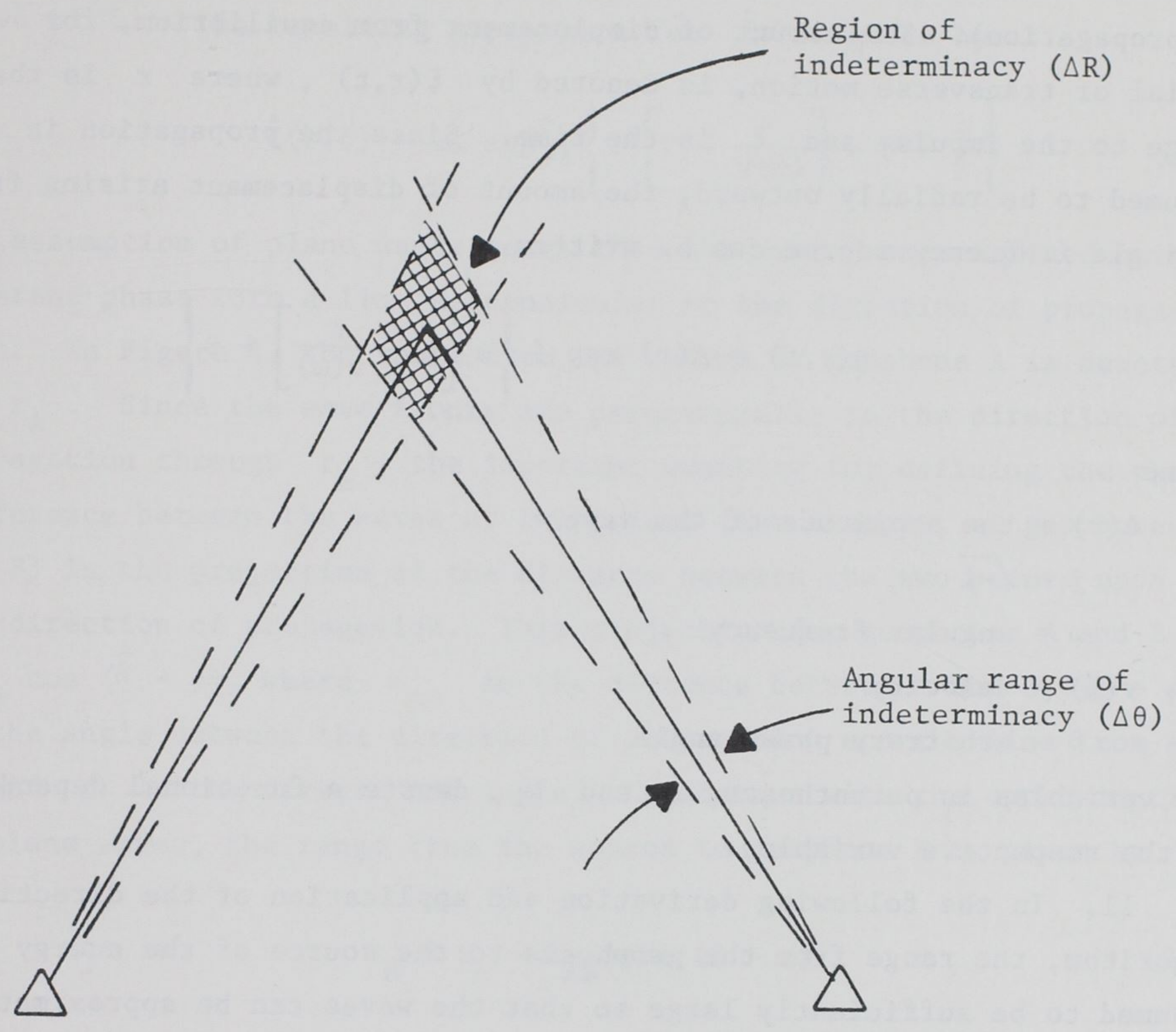

LEGEND

Region of indeterminacy $(\triangle \mathrm{R})$

$\triangle \quad$ Short-based array

Figure 3. Definition of region of indeterminacy 
from the impulse to the location at which the medium is observed. The displacement can be in the radial direction, or in either of the two transverse directions (i.e. displacements perpendicular to the direction of propagation). The amount of displacement from equilibrium, for radial or transverse motion, is denoted by $\xi(r, t)$, where $r$ is the range to the impulse and $t$ is the time. Since the propagation is assumed to be radially outward, the amount of displacement arising from a single frequency source can be written

$$
\xi(r, t)=A(r) \exp j\left\{\omega\left[t-\frac{r}{v(\omega)}\right]+\theta\right\}
$$

where

$$
\begin{aligned}
A(r) & =\text { the amplitude of the wave } \\
\mathrm{j} & =\sqrt{-1} \\
\omega & =\text { angular frequency } \\
\mathrm{v}(\omega) & =\text { velocity } \\
\theta & =\text { arbitrary phase angle }
\end{aligned}
$$

The variables in parentheses, $r$ and $\omega$, denote a functional dependence on the respective variables.

11. In the following derivation and application of the directional algorithm, the range from the geophones to the source of the energy is assumed to be sufficiently large so that the waves can be approximated as plane waves (i.e., the two-dimensional wave front defined by the spatial distribution of the wave having constant phase is spatially described by straight lines rather than by its actual circular shape). As shown in Appendix A, the important quantity for describing the accuracy of these assumptions is the ratio of the range $r$ to the geophone spacing $\&$ in the short-based array. Within the Remotely Monitored Battlefield Sensor System (REMBASS) minimum accuracy requirements, a ratio of 11 or greater is acceptable. Within the area contained inside the short-based array for which the plane wave approximation is valid, the amplitude of the wave can be assumed to be independent of the range. A rigorous treatment of the possible error arising from the plane wave assumption is presented in Appendix A. 
12. The plane waves approach the short-based array (geophones A, $B$, and $C$ ) as shown in Figure 4. The quantity sensed by the geophones is the particle velocity $\dot{\xi}$, which is obtained by taking the time derivative of $\xi$. The resulting expression for $\dot{\xi}$ is given by

$$
\dot{\xi}(r, t)=\omega A \exp j\left\{\omega\left[t-\frac{r}{v(\omega)}\right]+\theta+\frac{\pi}{2}\right\}
$$

The assumption of plane waves means that the wave fronts defined by a constant'phase form a line perpendicular to the direction of propagation. In Figure 4, the range from the source to geophone $A$ is denoted by $\mathrm{r}_{\mathrm{A}}$. Since the wave fronts are perpendicular to the direction of propagation through $\mathrm{r}_{\mathrm{A}}$, the important quantity for defining the phase difference between the waves at two points (for example, at geophones A and $B$ ) is the projection of the distance between the two points upon the direction of propagation. This projection for geophones $A$ and $B$ is ${ }_{A B} \cos (\beta-\phi)$, where $\ell_{A B}$ is the distance between $A$ and $B,(\beta-\phi)$ is the angle between the direction of propagation and the line from $A$ to $B$, and $\phi$ is the directional angle. Consistent with the approximation of plane waves, the range from the source to geophone $B$ is given by

$$
r_{B}=r_{A}-l_{A B} \cos (\beta-\phi)
$$

Similarly, the range from the source to $\mathrm{C}$ is given by

$$
r_{C}=r_{A}-\ell_{A C} \cos \phi
$$

Equations 2 and 3 are consistent with the plane wave approximation because their accuracy rests upon the approximation that the directions of propagation of the waves passing through all three geophones are the same. This is true only if the waves are accurately described as plane waves across the space of the array.

13. Equations 2 and 3 can be substituted into Equation 1 to obtain the expressions for particle velocity at geophones A, B, and C, as follows: 


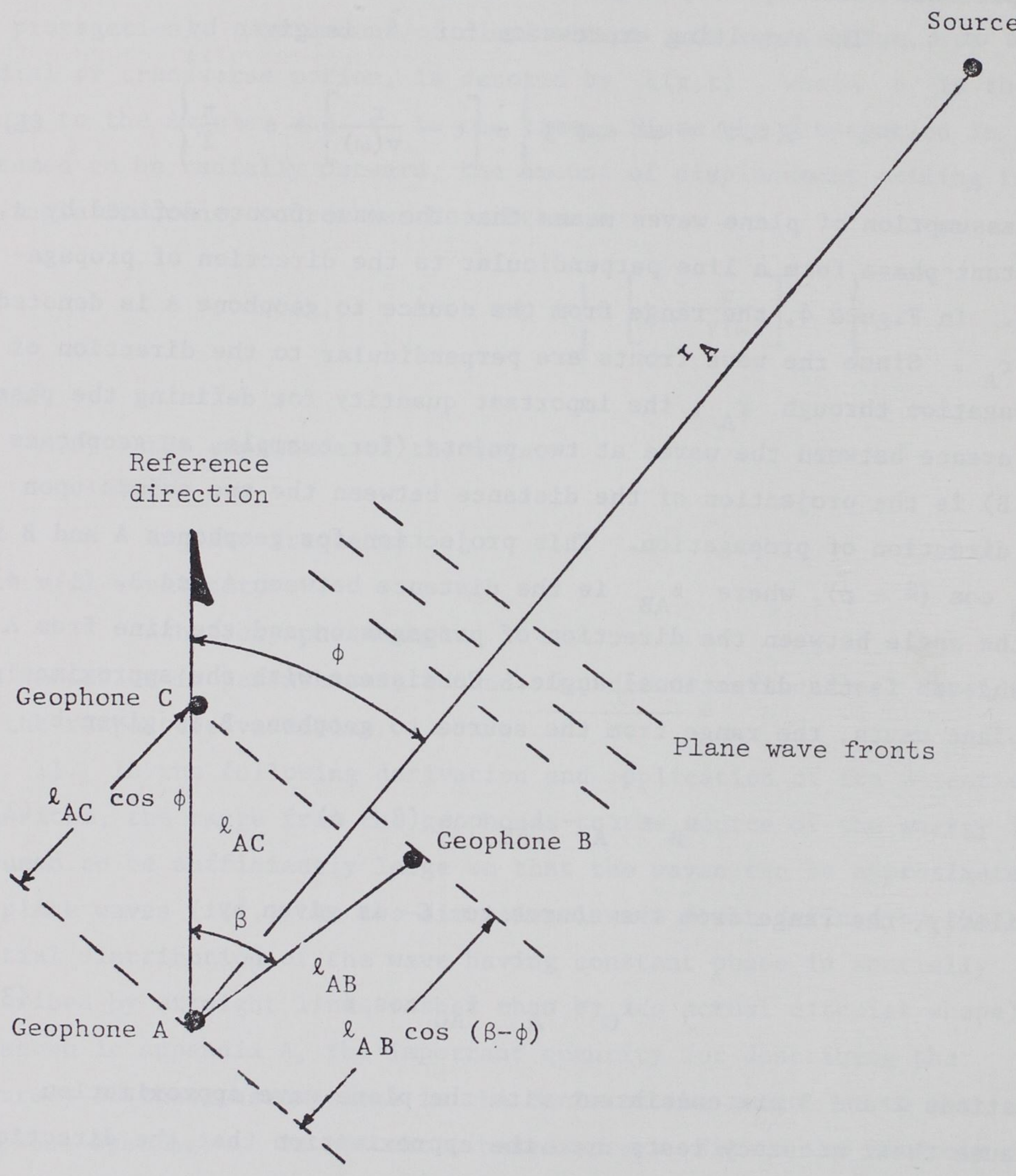

Figure 4. Geometric relation between sensors and incoming plane wave 


$$
\begin{aligned}
& \dot{\xi}_{A}=\omega A \exp j\left\{\omega\left[t-\frac{r_{A}}{v(\omega)}\right]+\theta+\frac{\pi}{2}\right\} \\
& \dot{\xi}_{B}=\omega A \exp j\left\{\omega\left[t-\frac{r_{A}-l_{A B} \cos (\beta-\phi)}{v(\omega)}\right]+\theta+\frac{\pi}{2}\right\} \\
& \dot{\xi}_{C}=\omega A \exp j\left\{\omega\left[t-\frac{r_{A}-l_{A C} \cos \phi}{v(\omega)}\right]+\theta+\frac{\pi}{2}\right\}
\end{aligned}
$$

Both Equation 4 and Equation 5 can be written in the form

$$
\dot{\xi}_{k=B, C}=\omega A \exp j\left\{\omega\left[t-\frac{r_{A}}{v(\omega)}\right]+\theta+\frac{\pi}{2}+\Delta \theta_{A k}\right\}
$$

where ${ }^{\Delta \theta}{ }_{A k}$ is the phase shift of the signatures at $B$ and $C$ relative to A. Therefore, the phase shifts are given by

$$
\Delta \theta_{A B}=\frac{-\omega l_{A B} \cos (\beta-\phi)}{v(\omega)}
$$

and

$$
\Delta \theta_{\mathrm{AC}}=\frac{-\omega \ell_{\mathrm{AC}} \cos \phi}{\mathrm{v}(\omega)}
$$

The time increment between the instant B receives the signal and the instant A receives the signal is given by

$$
\tau_{A B}=\frac{\ell_{A B} \cos (\beta-\phi)}{v(\omega)}
$$

Similarly, the time increment between the instant A receives the signal and the instant $C$ receives the signal is given by

$$
\tau_{\mathrm{AC}}=\frac{\ell_{\mathrm{AC}} \cos \phi}{\mathrm{v}(\omega)}
$$

Cos $(\beta-\phi)$ can be expanded using a trigonometric identity. The expanded form of Equation 8 can then be divided by Equation 9. The resulting expression for $\phi$ is found to be 


$$
\phi=\tan ^{-1}\left[\frac{1}{\sin \beta}\left(\frac{{ }^{l} A C^{\tau} A B}{{ }^{l} A B^{\top} A C}-\cos \beta\right)\right] \text { for } 0 \leq \phi \leq 360^{\circ}
$$

14. The spatial symmetry is optimized if the three geophones are placed at the vertices of an equilateral triangle. $\beta$ is then $60 \mathrm{deg}$ and Equation 10 reduces to

$$
\phi=\tan ^{-1}\left[\frac{2}{\sqrt{3}}\left(\frac{{ }^{\tau} \mathrm{AB}}{{ }^{\tau} \mathrm{AC}}-\frac{1}{2}\right)\right] \quad \text { for } 0 \leq \phi \leq 360^{\circ}
$$

Note that the signs of $\tau_{A B}$ and ${ }^{\tau} \mathrm{AC}$ must be used to determine the proper quadrant for $\phi$.

15. In the derivation of Equations 10 and 11 , a single frequency source was assumed. However, the forcing function of the source is, in general, impulsive in nature. Therefore, a wide band of frequencies is introduced into the medium. The particle velocity at the geophone is described by the continuous sum of all frequencies according to the expression

$$
\dot{\xi}(r, t)=\int_{-\infty}^{\infty} \omega A(\omega) \exp j\left\{\omega\left[t-\frac{r}{v(\omega)}\right]+\theta+\frac{\pi}{2}\right\} d \omega
$$

The signature can be processed, frequency by frequency, for directional estimates by obtaining ${ }^{\tau} A B$ and ${ }^{\tau} A C$ at each frequency. ${ }^{\tau} A B$ and ${ }^{\tau} A C$ are functions of frequency because the wave velocity is frequency dependent. However, as long as the frequency increments chosen for directional analysis are sufficiently narrow, such that the wave velocity in a given frequency increment can be considered to be constant, application of Equations 10 and 11 to obtain directional estimates is justified.

\section{Methods of Signature Analysis}

16. The signatures received by the geophones in the short-based array must be processed in some manner to obtain the time delays ${ }^{\tau} A B$ and $\tau_{\mathrm{AC}}$ (Equations 8 and 9). Equivalently, the phase shifts ${ }^{\Delta \theta}{ }_{\mathrm{AB}}$ and $\Delta \theta_{\mathrm{AC}}$ (Equations 6 and 7 ) can be obtained for each frequency $\omega$. 
As stated in paragraph 15, the signatures generally contain a broad spectrum of frequencies. The degree of difficulty in analyzing the signatures is roughly proportional to the width of the spectral band that is processed to obtain ${ }^{\tau} A B$ and ${ }_{A B}{ }_{A C}$. For seismic signatures of military targets, the spectral region of interest is generally between 0 and $400 \mathrm{~Hz}$. As the width of the spectral band being processed is narrowed, the capabilities of the signal-processing technique are enhanced. This fact becomes particularly apparent in multiple-target or noisy-background environments because the "targets" are 1ikely to transmit power differently for different spectral regions of the signature as indicated in Figure 5a for two targets ( 1 and 2). Since the signatures of targets 1 and 2 are superimposed, the power spectrum observed at the geophone array has the form indicated in Figure 5b. The superposition of the signatures of targets 1 and 2 presents a problem in interpreting the calculated time increments $\tau_{A B}$ and ${ }^{\tau_{A C}}$. In the shaded frequency region of the spectrum (Figure $5 \mathrm{~b}$ ), the signatures of both targets make approximately the same contributions to the spectrum and, therefore, have approximately equivalent "rights" to the resulting calculated value of $\tau_{A B}$ or ${ }^{A} A C \cdot$ Generally, the calculated directional angle will fall somewhere between the two targets. However, in the spectral regions where the power spectrum of target 1 dominates over that of target 2 , the calculated values of ${ }^{\tau} \mathrm{AB}$ and ${ }^{\tau} \mathrm{AC}$ result in accurate calculations of the direction to target 1 . In a practical situation, the observer has no way of knowing from the calculated directional angles alone which angles are accurate descriptors of the direction to the target(s). Other properties of the signatures that describe the degree of superposition of the waves must be invoked to aid in this decision, which adds an additional complexity to the signal-processing technique.

17. Although several techniques are available for signal processing, two techniques have been selected that have differing degrees of complexity and corresponding differences in their capabilities. The two techniques will be referred to as cross-correlation analysis and coherent-signature analysis. The cross-correlation analysis was considered because of its simplicity. However, because of the shortcomings 


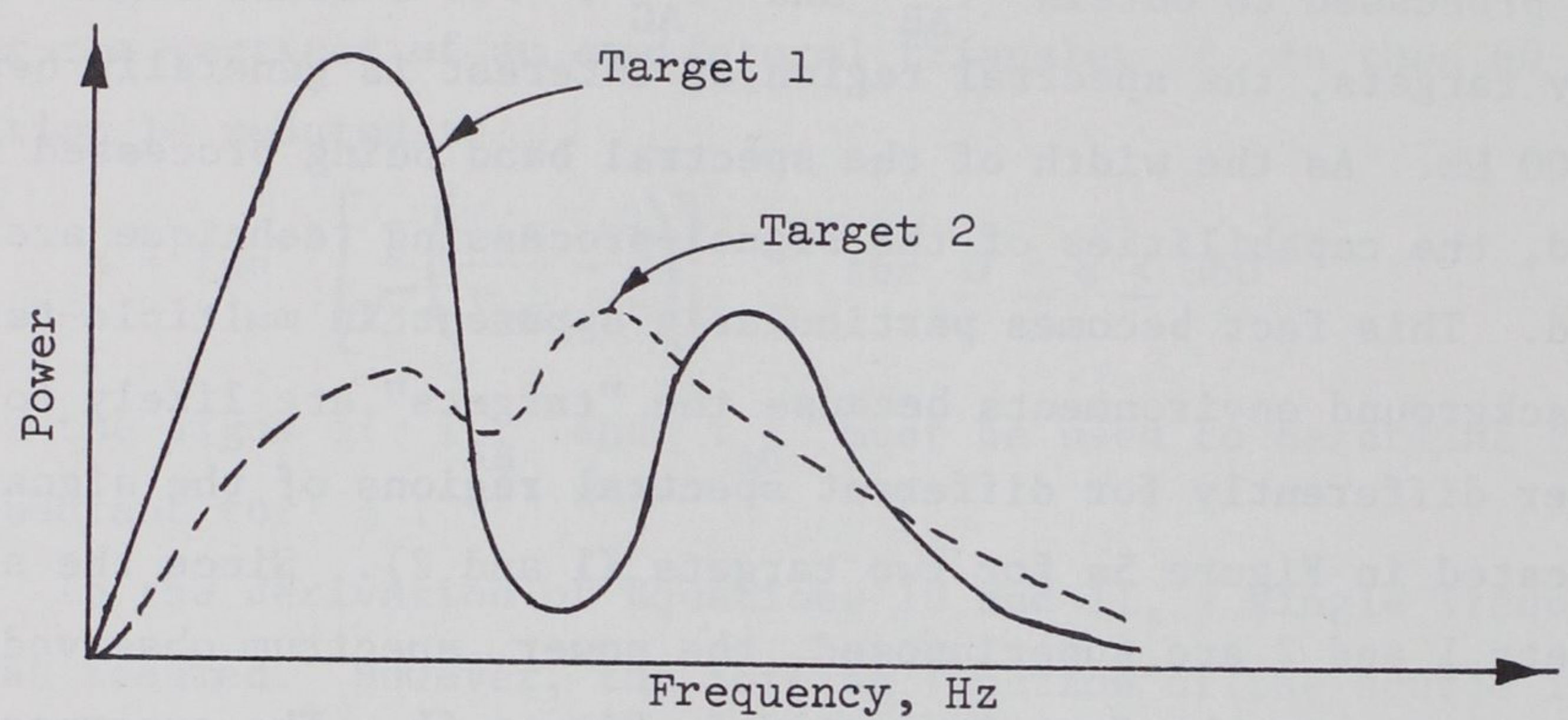

a. Power spectra for signatures emanating from two targets

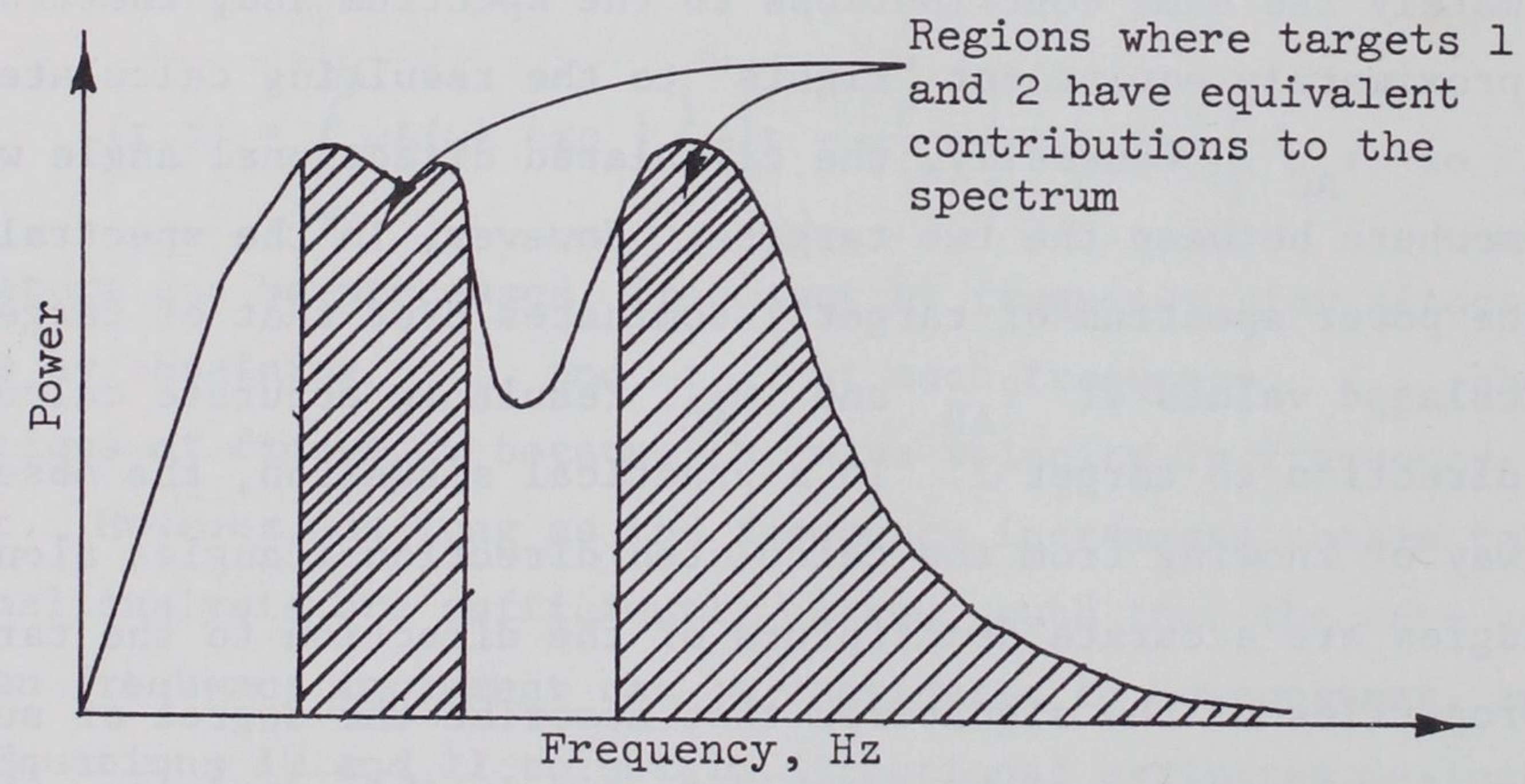

b. Power spectrum of signature at the array in a two-target (targets 1 and 2) environment

Figure 5. Contributions of signatures of two targets to the power spectrum observed at the array in a two-target environment 
of cross-correlation analysis (discussed later), the coherent-signature analysis was implemented. The coherent-signature analysis has a demonstrated applicability to processing of pressure waves in water for calculating directional angles using an array of pressure transducers.* The primary difference between the two analysis techniques (i.e. crosscorrelation and coherent-signature) is the frequency bands in which ${ }^{\tau} \mathrm{AB}$ and $\tau_{\mathrm{AC}}$ (Equations 8 and 9) are computed. The cross-correlation analysis yields values of $\tau_{A B}$ and $\tau_{A C}$ in the frequency region of maximum energy; the coherent-signature analysis results in calculated values for $\tau_{A B}$ and $\tau_{A C}$ in a number of narrow frequency bands.

18. It should be noted that the requirement for continuous signatures over time for coherent-signature analysis excludes its application, as implemented in this study, to impulsive targets such as artillery firing; however, cross-correlation analysis is applicable to impulsive signatures.

Cross-correlation analysis

19. Cross-correlation analysis is based on the cross-covariance estimate $* * \mathrm{C}_{i j}$ for a time shift $u$ between two signatures $\dot{\xi}_{i}$ and $\dot{\xi}_{j}$ where

$$
C_{i j}(u)=\frac{1}{T} \int_{-T / 2}^{T / 2} \dot{\xi}_{i}(t) \dot{\xi}_{j}(t+u) d t
$$

The cross-covariance is estimated for each pair of geophones $i$ and $j$ in the triangular array and for a series of time shifts $u$ such that $u$ is less than $T \cdot T$ is the length of the integration interval and must be greater than both the period of the dominant frequency band and the anticipated time delay between signatures, and $d t$ is an increment in the time domain. The times $\tau_{A B}$ and $\tau_{A C}$ are then obtained by finding the values of $u$ for which $C_{A B}$ and $C_{A C}$ are maximized. It should be emphasized that the times $\tau_{A B}$ and $\tau_{A C}$ are frequency dependent because

\footnotetext{
* Ibid., p. 8 .

** G. M. Jenkins and D. G. Watts, Spectral Analysis and Its Applications, Holden-Day, San Francisco, Calif., 1969.
} 
of dispersion, and the times obtained using cross-correlation analysis are characteristic of the frequencies of the dominant energy band. Coherent-signature analysis

20. In principle, it should be possible to compute directional angles for each frequency present in the seismic signature. In practice, however, direct analysis using a discrete Fourier transform of the raw data results in a scattering of the computed values of ${ }^{\tau} A B$ and ${ }^{\tau} \mathrm{AC}$ and a resulting scattering of the computed directional angles for each frequency. One approach to diminishing the amount of scattering is to average the $\tau_{\mathrm{AB}}$ and $\tau_{\mathrm{AC}}$ or parameters related to their computation (i.e. Fourier coefficients, etc.) over time. Although an averaging technique is incorporated into the coherent-signature analysis, it does not sufficiently reduce the scattering in the calculated values of ${ }^{{ }_{A B}}$ and $\tau_{A C}$, primarily because the length of the averaging time cannot be longer than several seconds for meaningful directional calculations to moving targets. Therefore, some criterion is needed that will separate the potentially accurate values of ${ }^{\tau}{ }_{A B}$ and ${ }^{\tau}{ }_{A C}$ from the remaining ones. The property of the signatures that describes the consistency in the calculated phase shifts between the geophones in the array has proven useful in evaluating the accuracy of the computed value of ${ }^{\tau} A B$ and $\tau_{\mathrm{AC}}$ - The parameter associated with this property of the signatures is the coherence.* The coherence estimate, as determined for the signature analysis reported subsequently, has the following properties:

a. If the power of the signature remains reasonably constant at a given frequency during the signature time required for estimating coherence (approximately $2 \mathrm{sec}$ ), and the phase shift remains constant, the coherence is approximately unity.

b. If the phase shift changes with time, the value of the coherence becomes progressively smaller with increases in the magnitude of the time-dependent changes in phase shift and the length of the averaging time.

It seems reasonable to assume that for continuous seismic signatures emanating from a target, the phase shift should be reasonably consistent over relatively short time intervals (approximately $2 \mathrm{sec}$ ) and, therefore,

* Ibid., p. 21. 
a correlation should exist between a coherence estimate near unity and an accurate value of the calculated directional angle. This correlation is the basis for using the coherence parameter to evaluate the accuracy of the calculated directional angles at each frequency.

\section{Sources of Error in Calculating Target Direction}

21. Application of the directional algorithm, Equation 11, is susceptible to several sources of error. Generally, these errors can be separated into two categories: (a) instrumentation and signalprocessing errors, and (b) wave propagation errors. Errors in the first category arise from phase response characteristics of the instrumentation, finite spatial size of the geophones (i.e., they do not sample a point in space as assumed in the derivation of the directional algorithm), and numerical errors of a statistical nature that occur within the signature processing techniques. Errors in the second category arise from refraction and reflections of the seismic wave on its path from the target to the geophones and from errors in the plane wave approximation (paragraph 11). These errors are further treated in Appendix A. An additional potential source of error arises from the difficulty in proper interpretation of the times $\tau_{\mathrm{AB}}$ and $\tau_{\mathrm{AC}}$ from the calculated phase shifts $\Delta \theta_{\mathrm{AB}}$ and ${ }^{\Delta \theta_{\mathrm{AC}}}$ (Equations 6 and 7). The coherent-signature analysis techniques (paragraph 20) mathematically constrain the magnitude of the calculated phase shifts between $-\pi$ and $\pi$, even though the actual phase shift may be the calculated value $+2 \mathrm{n} \pi$ or $-2 \mathrm{n} \pi$, where $\mathrm{n}$ is an integer. This mathematical ambiguity of the true phase shift is an expression of a basic physical difficulty. In Figure 6, two sinusoidal time series are presented that are shifted in phase relative to each other. The question is: Which peaks in one series are associated with which peaks in the other? The ambiguity problem can be eased by shortening the distance between the geophones in the array. However, an ambiguity will always occur when the magnitude of the phase shift exceeds $\pi$. Since the phase shift is proportional to the frequency (Equation 6), there exists a cutoff frequency 
above which the ambiguity problem always occurs. The ambiguity can be removed for frequencies greater than the cutoff frequency by adding or subtracting $2 n \pi$ to the magnitude of the calculated phase shift, where $n$ is the appropriate integer.

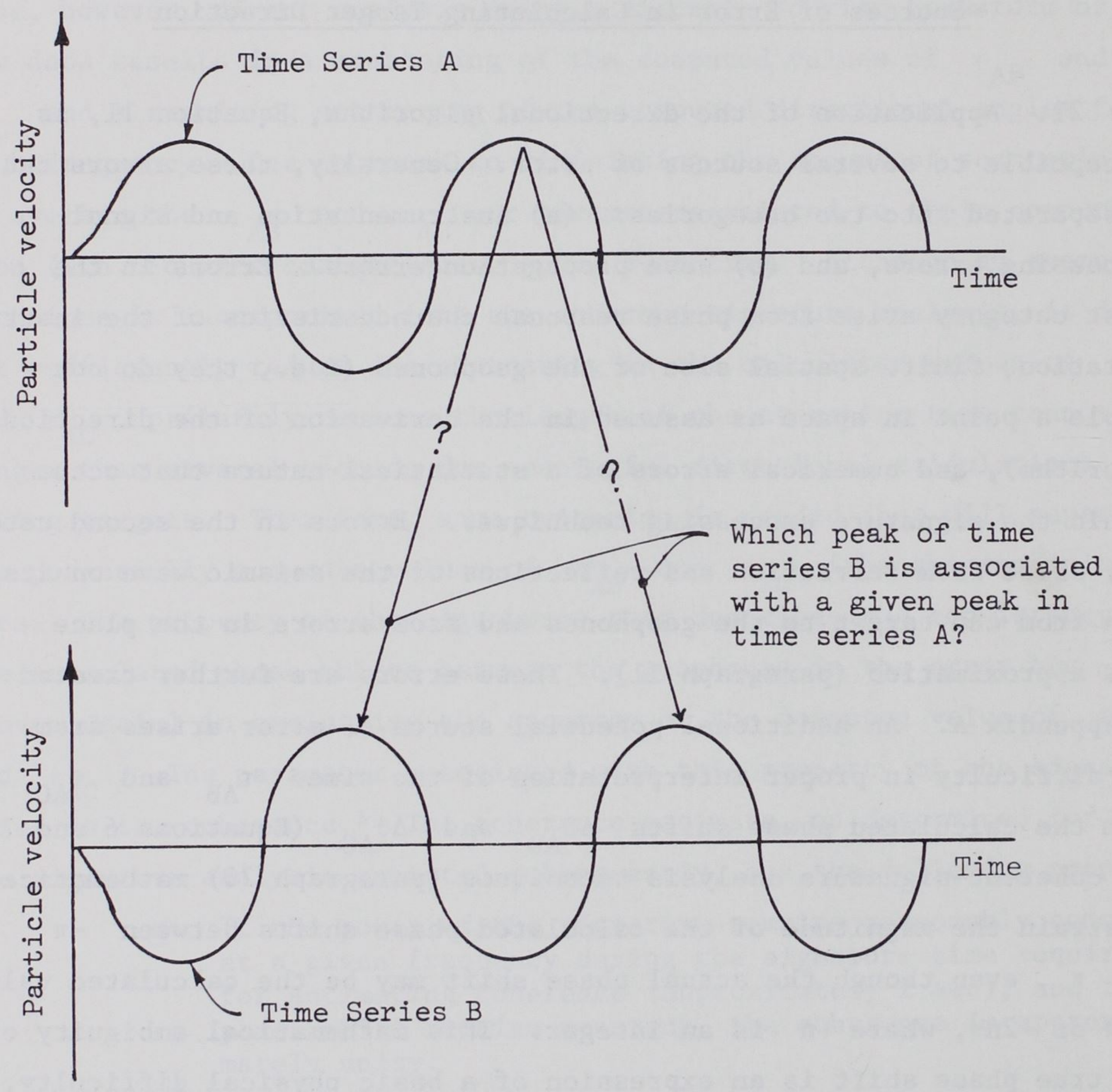

Figure 6. Comparison of two time series shifted in phase relative to each 
PART III: APPLICATION OF SIGNAL-PROCESSING

TECHNIQUES TO SIGNATURES OF MOVING TARGETS

\section{Data Collection}

22. Two test programs were conducted to obtain data for evaluating the seismic target-location system. The instrumentation and geophonearray deployment were tested in two geologically dissimilar test sites, and the results were subjected to analysis by the techniques described in paragraphs 19 and 20.

23. The first test program was conducted in January 1974 at Vicksburg, Mississippi. The second was conducted in April 1974 at Fort Carson, Colorado. The tests were designed around the following variables: vehicle type (i.e. M35, M113, M151, and M728), vehicle velocity, vehicle number (i.e. one or two vehicles moving simultaneously), surface condition (i.e. on-road or cross-country), range, geological conditions (i.e. Vicksburg and Fort Carson test sites), array spacing (2 $\mathrm{m}$ and $6 \mathrm{~m}$ ), and geophone type (Scientific HS-10 and Mark Products L-4 geophones). The configuration of each of the short-based arrays used in these two test programs is presented in Figure 7. Test variables for both test programs are tabulated in Appendix C.

\section{Vickburg tests}

24. The Vicksburg test site consisted of deep lean clay (loess) soils with a water table about $30 \mathrm{~cm}$ below the surface. Vehicles tested were an M113 APC (1ight tracked vehicle), an M35 (2-1/2-ton truck), and an M151 (jeep). The layout of the Vicksburg test site is shown in Figure 8. The geophone array, denoted by the triangular symbol, consisted of six vertical Geospace HS-10 geophones. Each geophone was implanted deep enough to allow its upper surface to be covered by several centimetres of top soil to reduce the possibility of acoustic signals coupling with the geophones. The data from each geophone were simultaneously recorded on a 14-track tape recorder at tape speeds of $3-3 / 4$ and $7-1 / 2$ ips. At either tape speed, the frequency response of the recorder is well above $1000 \mathrm{~Hz}$. The HS-10 geophone is 


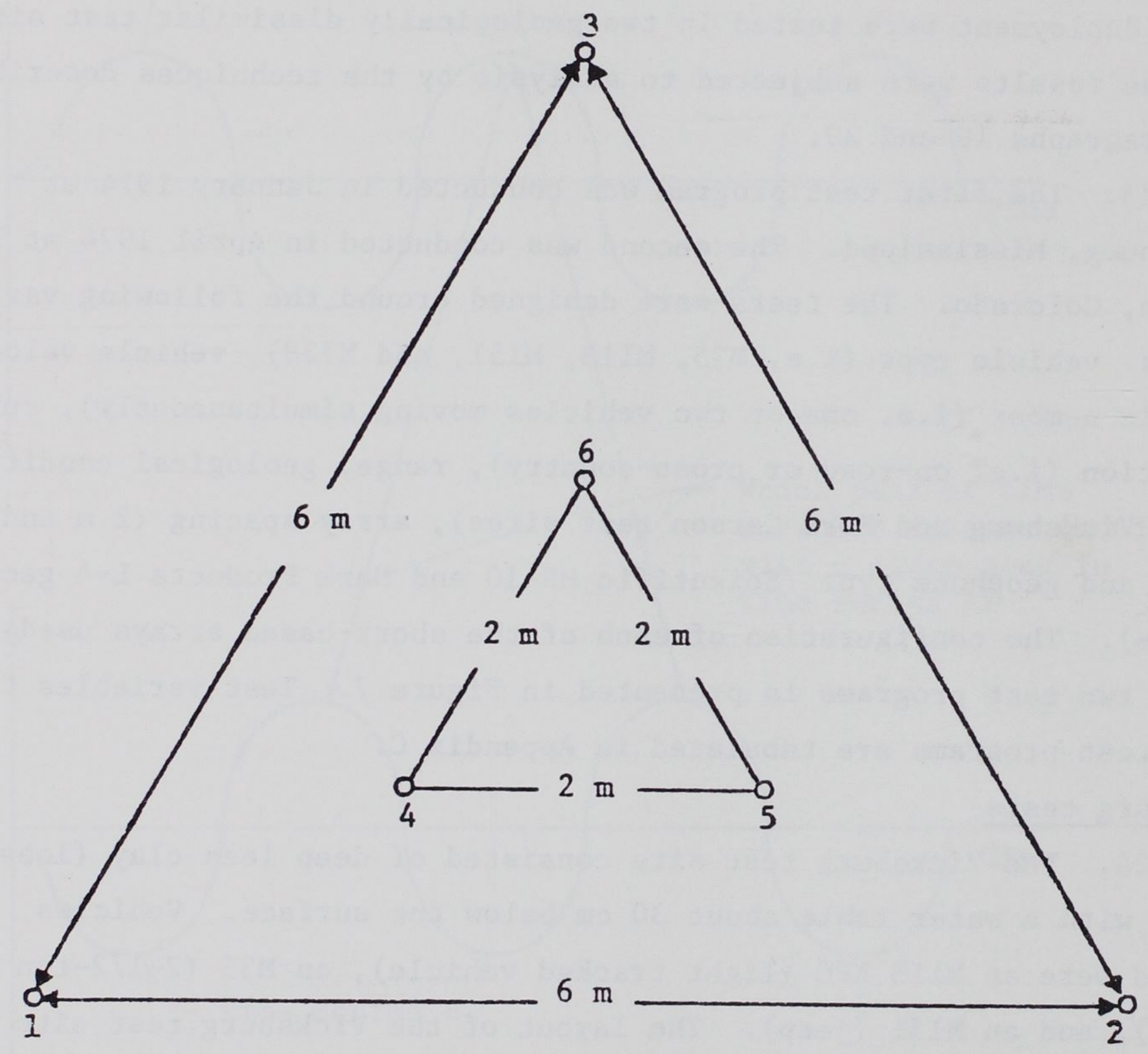

Figure 7. Geophone arrays and dimensions with geophones identified by numbers 1-6 


\section{LEGEND}

$\triangle$ Geophone array

$\square$ Vehicle

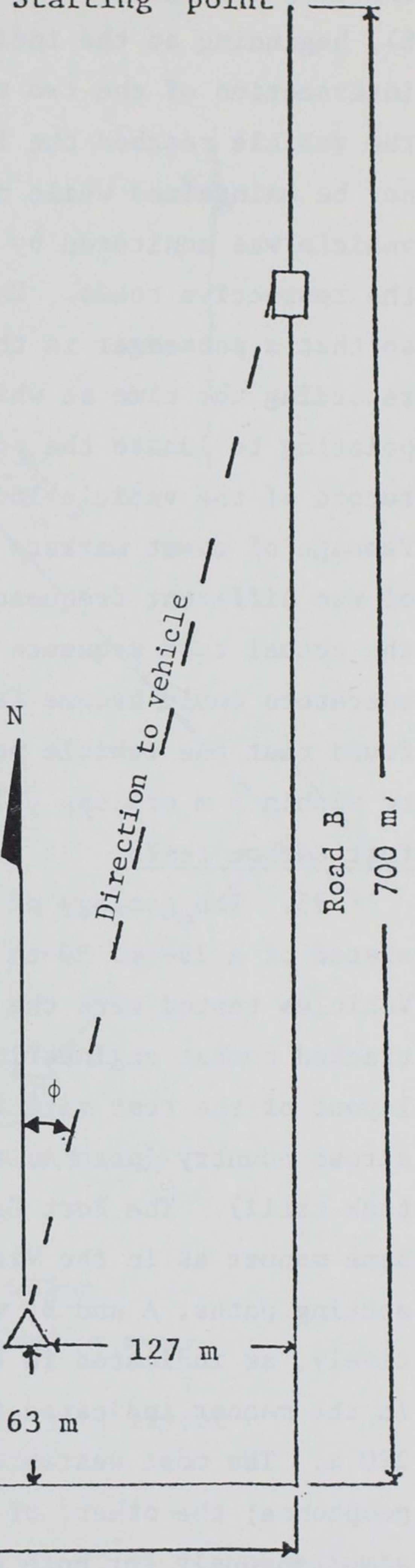

Figure 8. Vicksburg test site layout 
sensitive to frequencies from $1 \mathrm{~Hz}$ to somewhat greater than $350 \mathrm{~Hz}$. In the single-target tests, the vehicles were run on roads $A$ and $B$ (Figure 8 ), beginning at the indicated starting points and proceeding to the intersection of the two roads. Each test was terminated slightly before the vehicle reached the intersection because a constant velocity could not be maintained while negotiating the turn. The position of the vehicle was monitored by placing event markers at 50-m intervals along the respective roads. Radio contact was maintained during each test run so that a passenger in the vehicle could identify the location. By recording the time at which each event marker was passed, and interpolating to locate the position between event markers, an accurate record of the vehicle location was obtained throughout the test. Passage of event markers was recorded for each vehicle over radio links of two different frequencies. A number of trial runs were made prior to the actual test sequence so that drivers, passengers, and instrument operators could become familiar with the recording procedure. It was found that the vehicle position at the stakes could be easily monitored to within $5 \mathrm{~m}$ or less using the radio communication link.

\section{Fort Carson tests}

25. The geology of the Fort Carson test site was complex. It consisted of a 20- to 30-cm layer of silt overlying fractured limestone. Vehicles tested were the M113 APC, the M35, the M151, and an M728 (heavy tracked combat engineering vehicle mounted on an M60 chassis). The layout of the test site is shown in Figure 9. The vehicles were run across country (path A) and on a compacted surface (path B, a well-used tank trail). The Fort Carson tests were conducted in essentially the same manner as in the Vicksburg tests. Vehicles were run on two intersecting paths, $A$ and $B$, with straight-line runs of 500 and $550 \mathrm{~m}$, respectively, as indicated in Figure 9. Two arrays of geophones were deployed in the manner indicated in Figure 7 with a spacing between the arrays of $220 \mathrm{~m}$. The most westward array consisted of triaxial Mark Product L-4 geophones; the other, of Scientific HS-10 geophones. Data were recorded simultaneously for both arrays. 


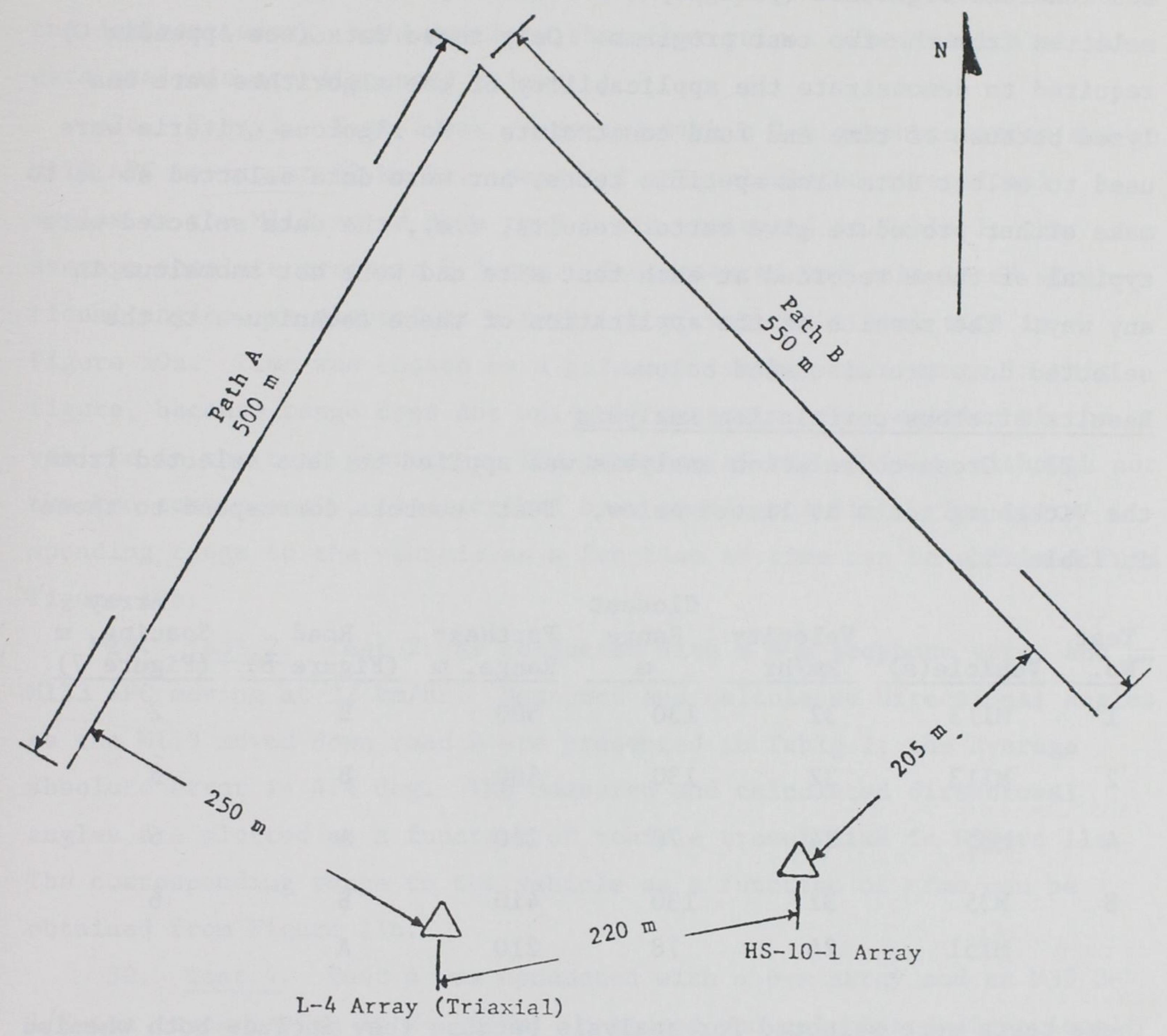

$\triangle \frac{\text { LEGEND }}{\text { Geophone array }}$

Figure 9. Fort Carson vehicle test site layout 


\section{Results of Signature Analysis}

26. The two techniques of signature analysis, cross-correlation and coherent-signature (paragraphs 19 and 20), were applied to data selected from the two test programs. Only those data (see Appendix C) required to demonstrate the applicability of the algorithms were analyzed because of time and fund constraints. No rigorous criteria were used to select data from specific tests, nor were data selected so as to make either procedure give better results, i.e., the data selected were typical of those recorded at each test site and were not anomalous in any way. The results of the application of these techniques to the selected data are discussed below.

Results of cross-correlation analysis

27. Cross-correlation analysis was applied to data selected from the Vicksburg tests as listed below. Test numbers correspond to those in Table $\mathrm{Cl}$.

\begin{tabular}{|c|c|c|c|c|c|c|}
\hline $\begin{array}{l}\text { Test } \\
\text { No. } \\
\end{array}$ & Vehicle(s) & $\begin{array}{l}\text { Velocity } \\
\mathrm{km} / \mathrm{hr} \\
\end{array}$ & $\begin{array}{c}\text { Closest } \\
\text { Range } \\
\mathrm{m} \\
\end{array}$ & $\begin{array}{l}\text { Farthest } \\
\text { Range, m }\end{array}$ & $\begin{array}{c}\text { Road } \\
\text { (Figure 8) } \\
\end{array}$ & $\begin{array}{c}\text { Array } \\
\text { Spacing, m } \\
\text { (Figure 7) }\end{array}$ \\
\hline 1 & M113 & 32 & 130 & 600 & B & 2 \\
\hline 2 & M113 & 32 & 130 & 600 & B & 6 \\
\hline 4 & M35 & 24 & 70 & 280 & A & 6 \\
\hline \multirow[t]{2}{*}{8} & M35 & 32 & 130 & 410 & B & 6 \\
\hline & M151 & 24 & 78 & 210 & A & \\
\hline
\end{tabular}

These tests were selected for analysis because they include both wheeled and tracked vehicles traveling at representative velocities and usefully distant ranges, in addition to a multiple-target test. Directional angles were calculated at various ranges between the range bounds identified in the above tabulation. The vehicles were "tracked" along their respective roads ( $A$ and $B$ in Figure 8). Data were analyzed for the M113 tests using both the 2- and 6-m geophone arrays identified in Figure 7. In each of the tests, the seismic signatures were digitized at a rate of 1500 samples/sec. The continuous integral of Equation 12 
reduces to a discrete summation over the range of integration (i.e. $-\mathrm{T} / 2$ to $T / 2)$. The increment in the time domain, $d t$, is then given by $(1 / 1500)$ sec. An integration range defined by $T=0.2 \mathrm{sec}$ was found to be acceptable for analysis because the period of the seismic waves in the dominant energy band was less than that value. The results of the data analysis are discussed below.

28. Test 1. Test 1 was conducted with a 2-m geophone array and an M113 APC moving at $32 \mathrm{~km} / \mathrm{hr}$. Measured and calculated directional angles as the M113 moved down road B are presented in Table 1; the average absolute error is $8.3 \mathrm{deg}$. The measured and calculated directional angles are plotted as a function of vehicle travel time in Figure 10a. Time was chosen as a parameter rather than range in this figure, because range does not uniquely determine the position of the vehicle in the test geometry. The position of the vehicle, although not tracked continuously, is described by a continuous line. The corresponding range to the vehicle as a function of time can be obtained from Figure 10b.

29. Test 2. Test 2 was conducted with a 6-m geophone array and an M113 APC moving at $32 \mathrm{~km} / \mathrm{hr}$. Measured and calculated directional angles as the M113 moved down road B are presented in Table 1; the average absolute error is $4.4 \mathrm{deg}$. The measured and calculated directional angles are plotted as a function of vehicle travel time in Figure 11 . The corresponding range to the vehicle as a function of time can be obtained from Figure 11b.

30. Test 4. Test 4 was conducted with a 6-m array and an M35 2$1 / 2$-ton truck moving at $24 \mathrm{~km} / \mathrm{hr}$. Measured and calculated directional angles as the M35 moved down road A are presented in Table 2; the average absolute error is $4.7 \mathrm{deg}$. The measured and calculated directional angles are plotted as a function of vehicle travel time in Figure 12a. The corresponding range to the vehicle as a function of time can be obtained from Figure $12 b$.

31. Test 8 . Test 8 was conducted with a 6-m array and two vehicles, an M35 truck and an $\mathrm{N} 151$ jeep moving at 32 and $24 \mathrm{~km} / \mathrm{hr}$, respectively. Measured and calculated directional angles as the M35 moved 


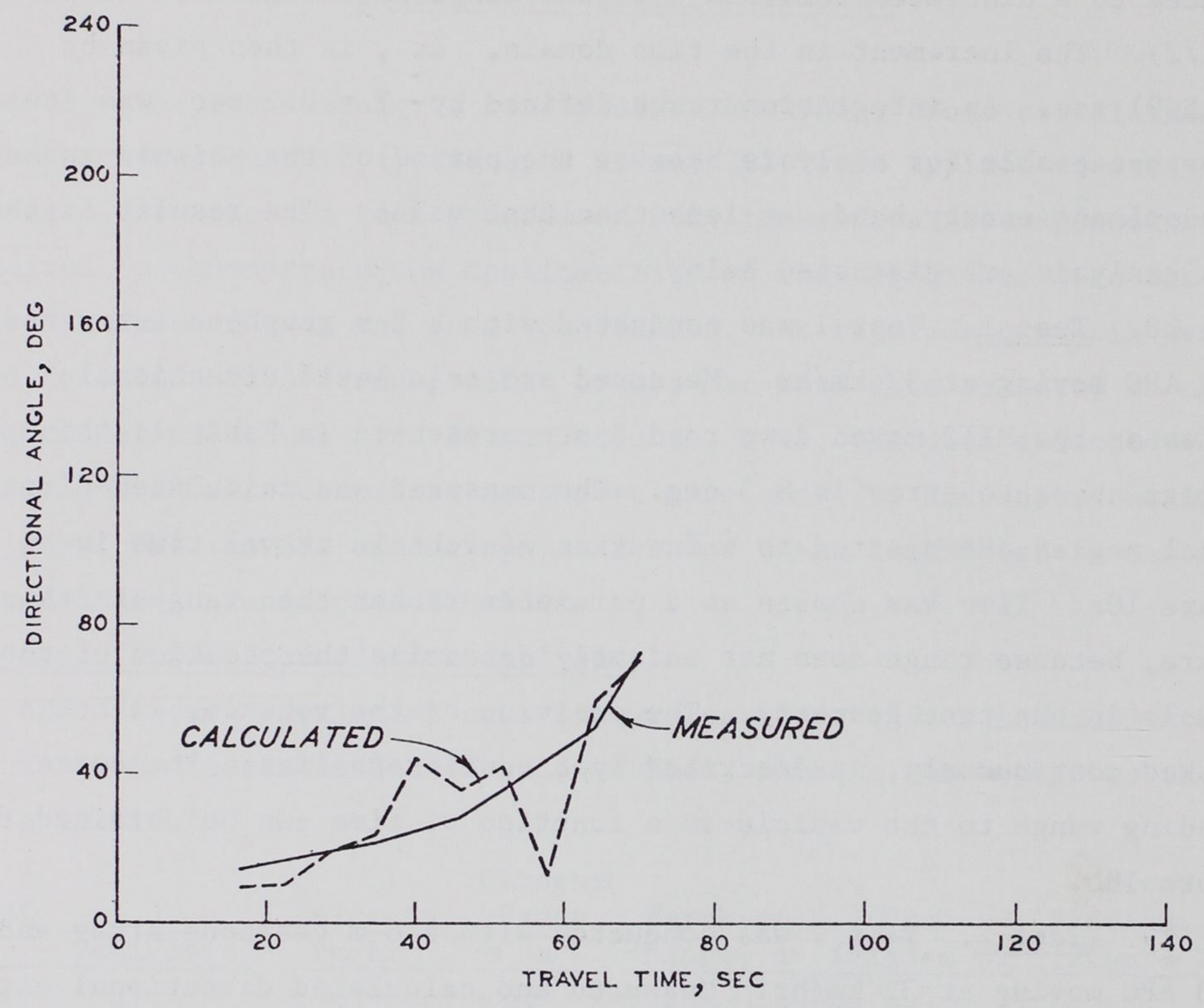

a. CALCULATED AND MEASURED DIRECTIONAL ANGLES

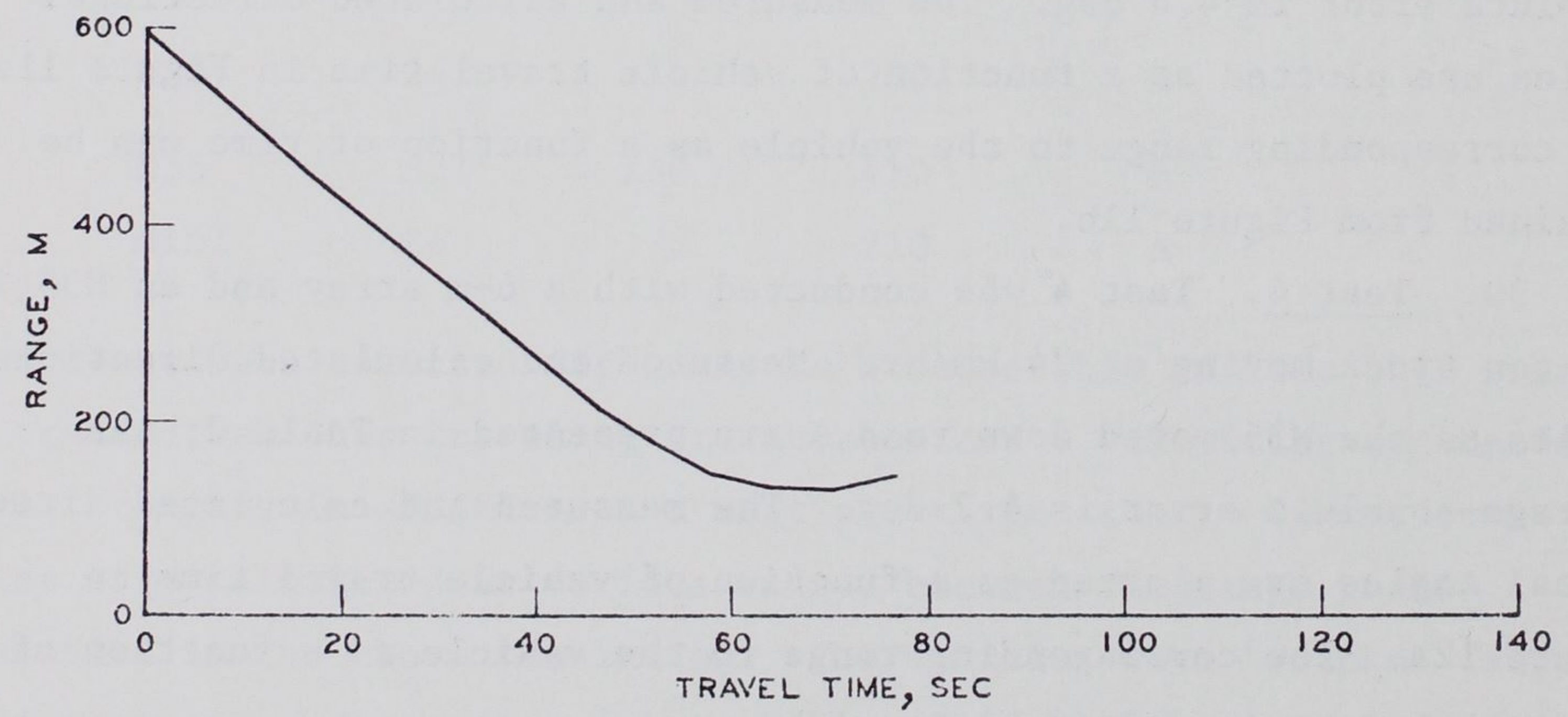

b. MEASUPED RANGE FROM SENSOR ARRAY TO TARGET

Figure 10. Position information for an Ml13 APC (velocity

$=32 \mathrm{~km} / \mathrm{hr}$, geophone spacing $=2 \mathrm{~m}$ ) using geophones 4,5 , and 6 (Figure 7) 


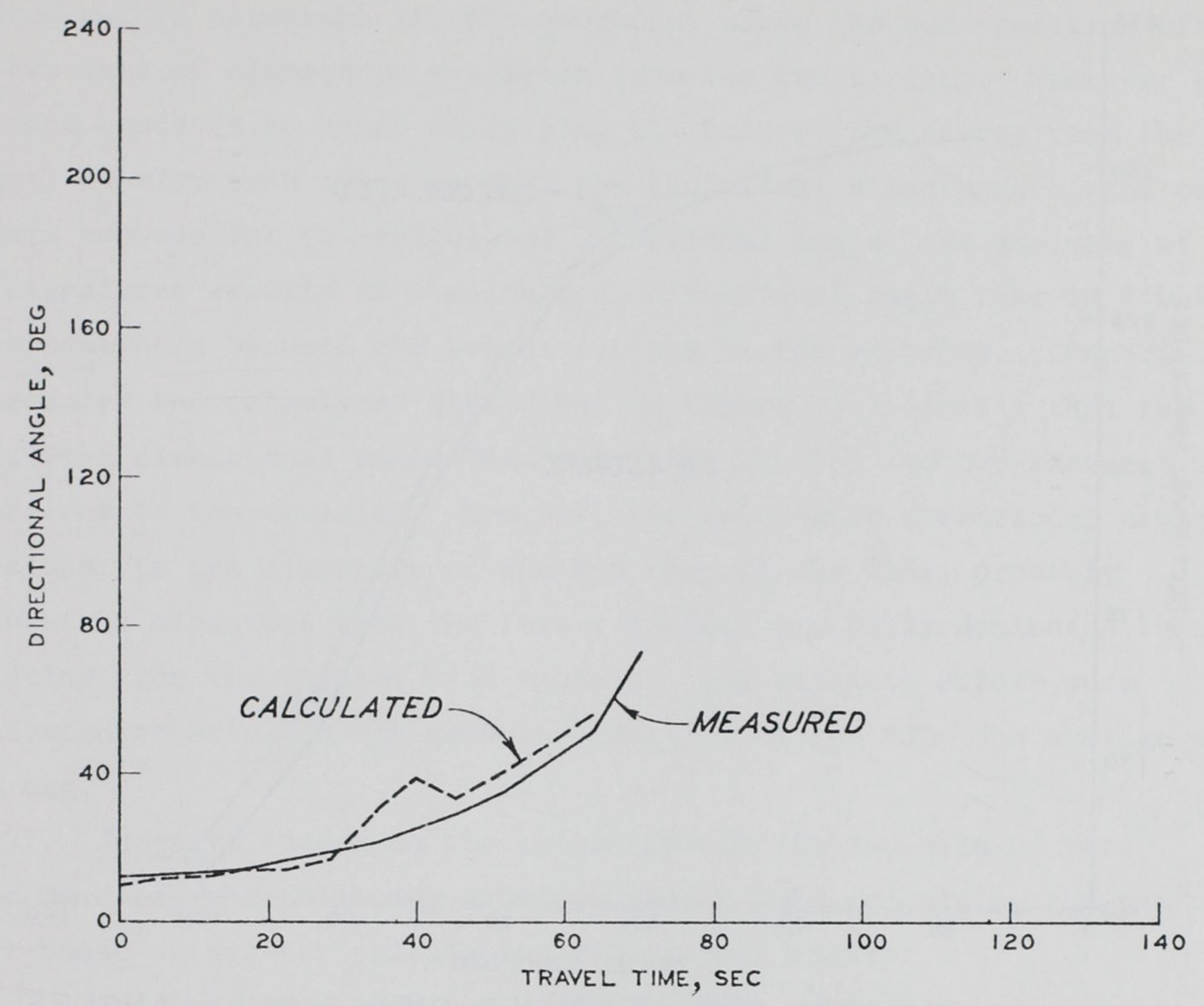

a. CALCULATED AND MEASURED DIRECTIONAL ANGLES

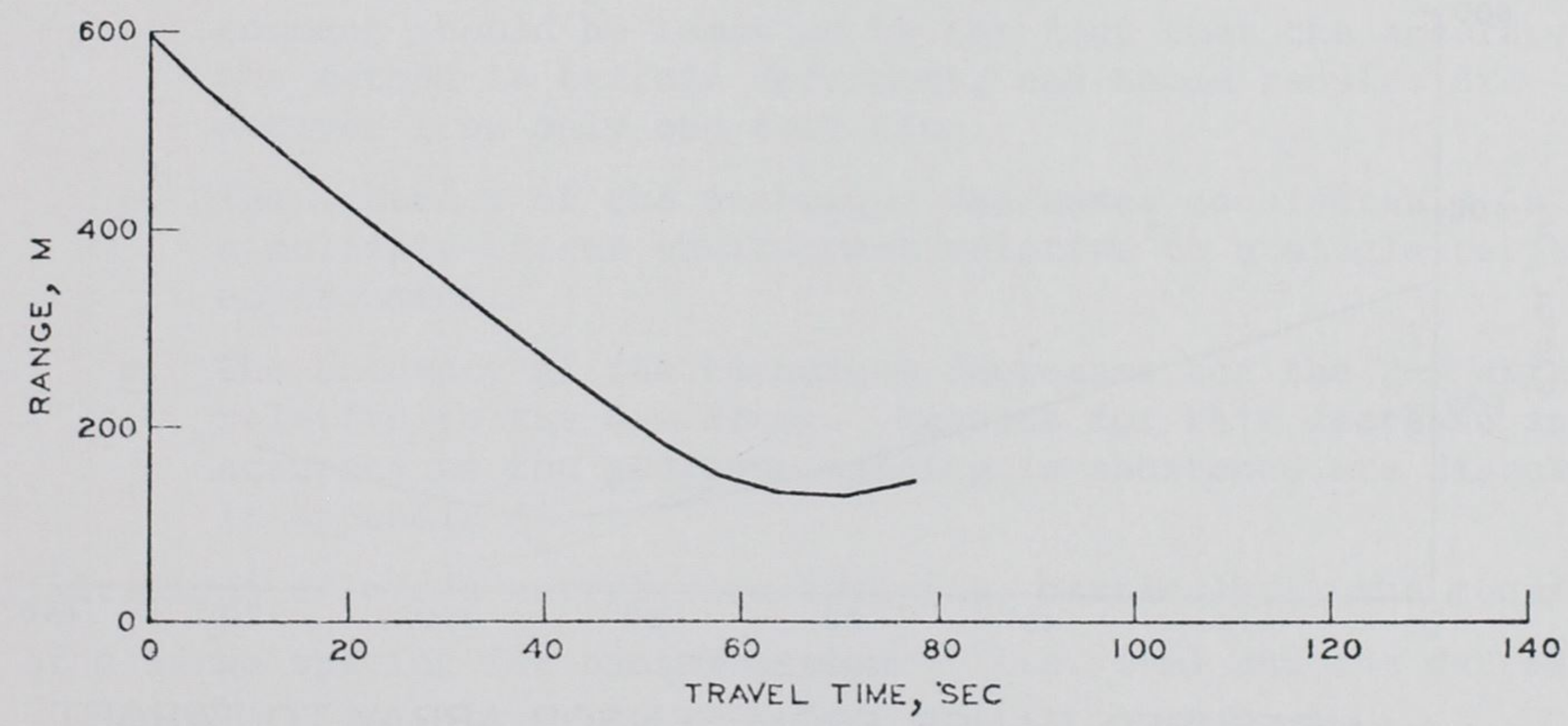

b. MEASURED RANGE FROM SENSOR ARRAY TO TARGET

Figure 11. Position information for an Ml13 APC (velocity

$=32 \mathrm{~km} / \mathrm{hr}$, geophone spacing $=6 \mathrm{~m}$ ) using geophones 1,2 , and 3 (Figure 7) 


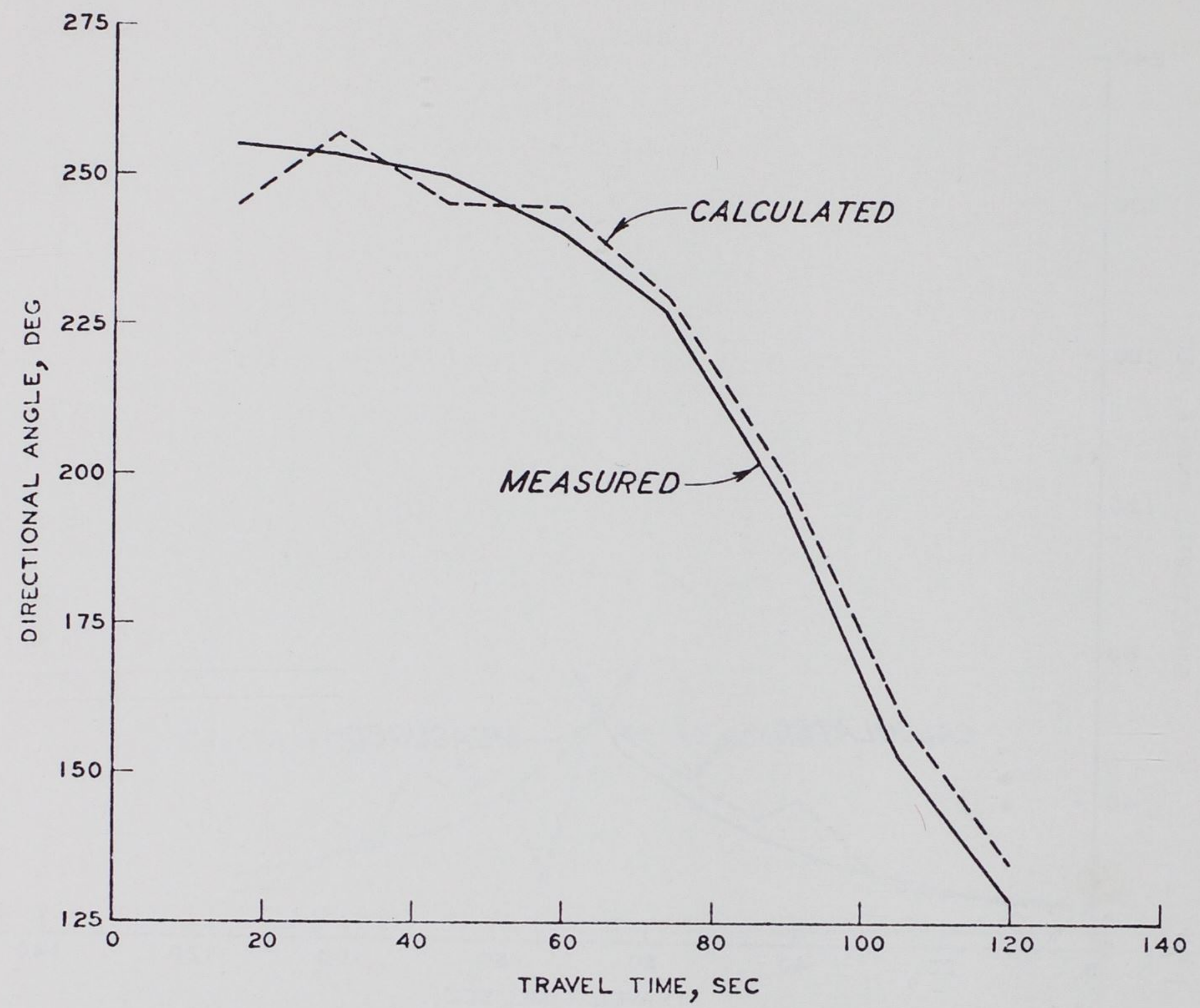

a. CALCUL ATED AND MEASURED DIRECTIONAL ANGLES

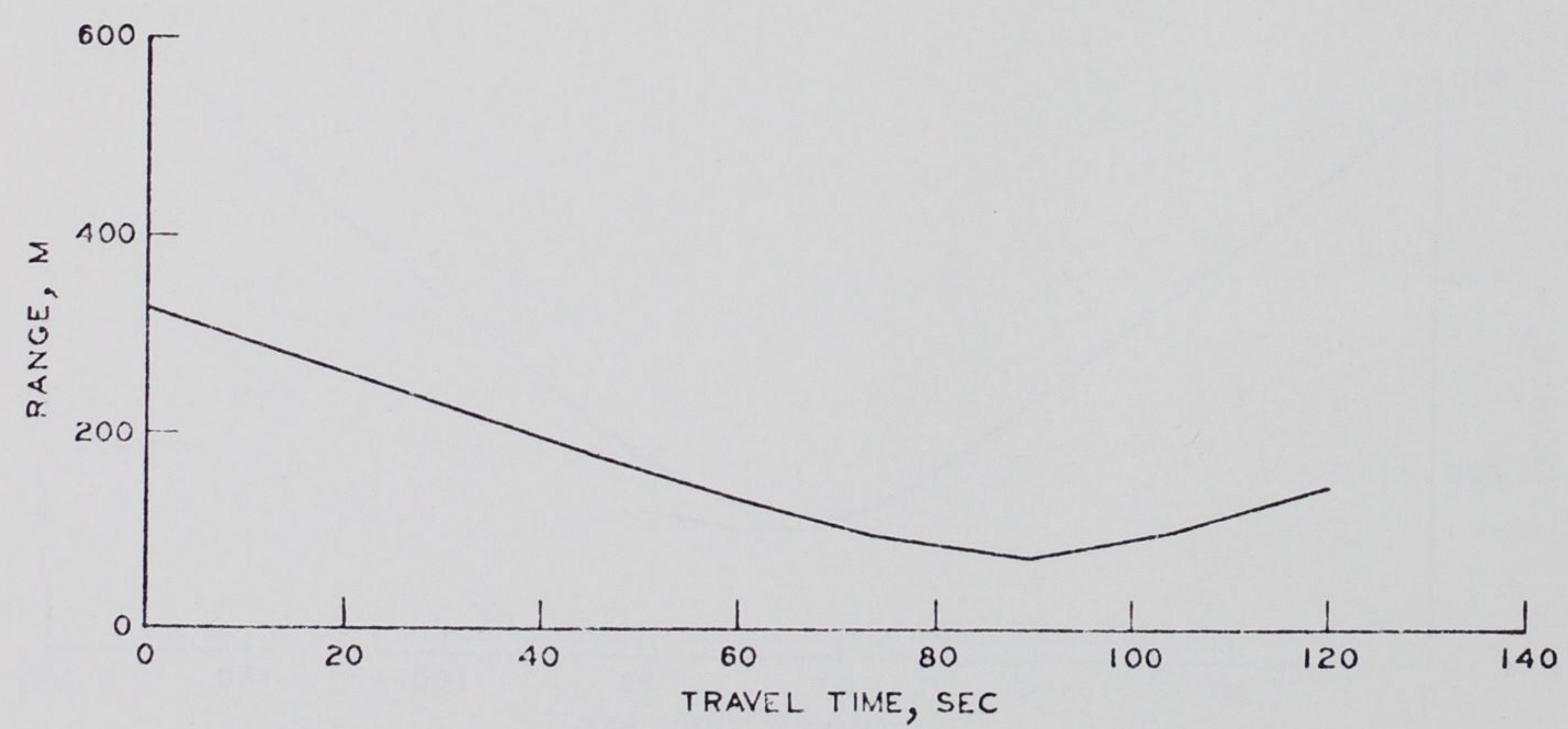

b. MEASURED RANGE FROM SENSOR ARRAY TO TARGET

Figure 12. Position information for an M35 truck (velocity

$=24 \mathrm{~km} / \mathrm{hr}$, geophone spacing $=6 \mathrm{~m}$ ) using geophones 1,2 , and 3 (Figure 7) 
down road B and the M151 moved down road A are presented in Table 3 . As pointed out in paragraph 16, the geophones sense the superposition of the two sets of signatures emanating from the two targets. Whenever the dominant bands (i.e. bands containing the bulk of the energy from the target) overlay each other so that the individual signatures of the two targets compete for the calculated directional angle, the analysis of the signatures results in a calculated directional angle that is likely to be somewhere between the two directions to the vehicles. Comparison of measured and calculated directions in Figure 13 indicates that the calculated directional angles lie generally between the two measured directions to the vehicles. However, the calculated directional angles are closer to the direction of the M35 than of the M151, probably because the signature from the former vehicle generally dominated that emanating from the 1ighter M151 vehicle. The absolute errors were calculated relative to the measured location of the M35; the average was $20.3 \mathrm{deg}$.

32. Summary. Based on the cross-correlation analysis of the selected data, the following comments can be made concerning the use of short-based arrays for calculating directional angles:

a. The array with $6-\mathrm{m}$ spacing can be used to obtain directional angles within an average accuracy of less than $5 \mathrm{deg}$ in a single-target (or quiet-background) environment. This comment should be tempered by the fact that the accuracy of the method is terrain dependent, and these results are derived from only one test site.

b. The accuracy of the technique decreases considerably in a multiple-target environment relative to a single-target environment.

c. The accuracy of the technique decreases for the 2-m array relative to the 6-m array. Reasons for this decrease in accuracy as the geophone spacing is shortened are discussed in Appendix A.

The limitations of cross-correlation analysis, particularly the requirement of a large spacing for useful accuracy (i.e. 6-m) and the decrease 


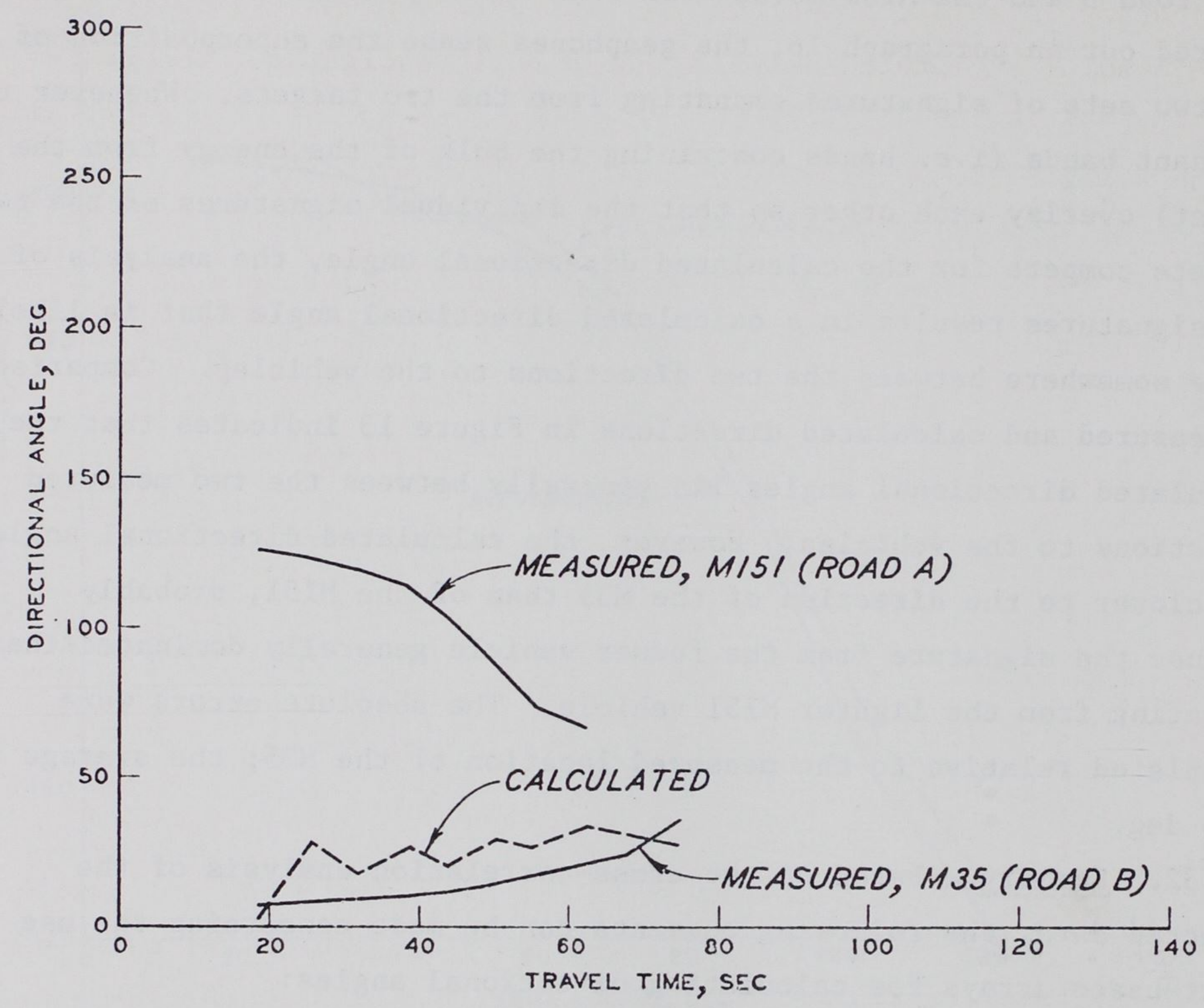

a. CALCULATED AND MEASURED DIRECTIONAL ANGLES

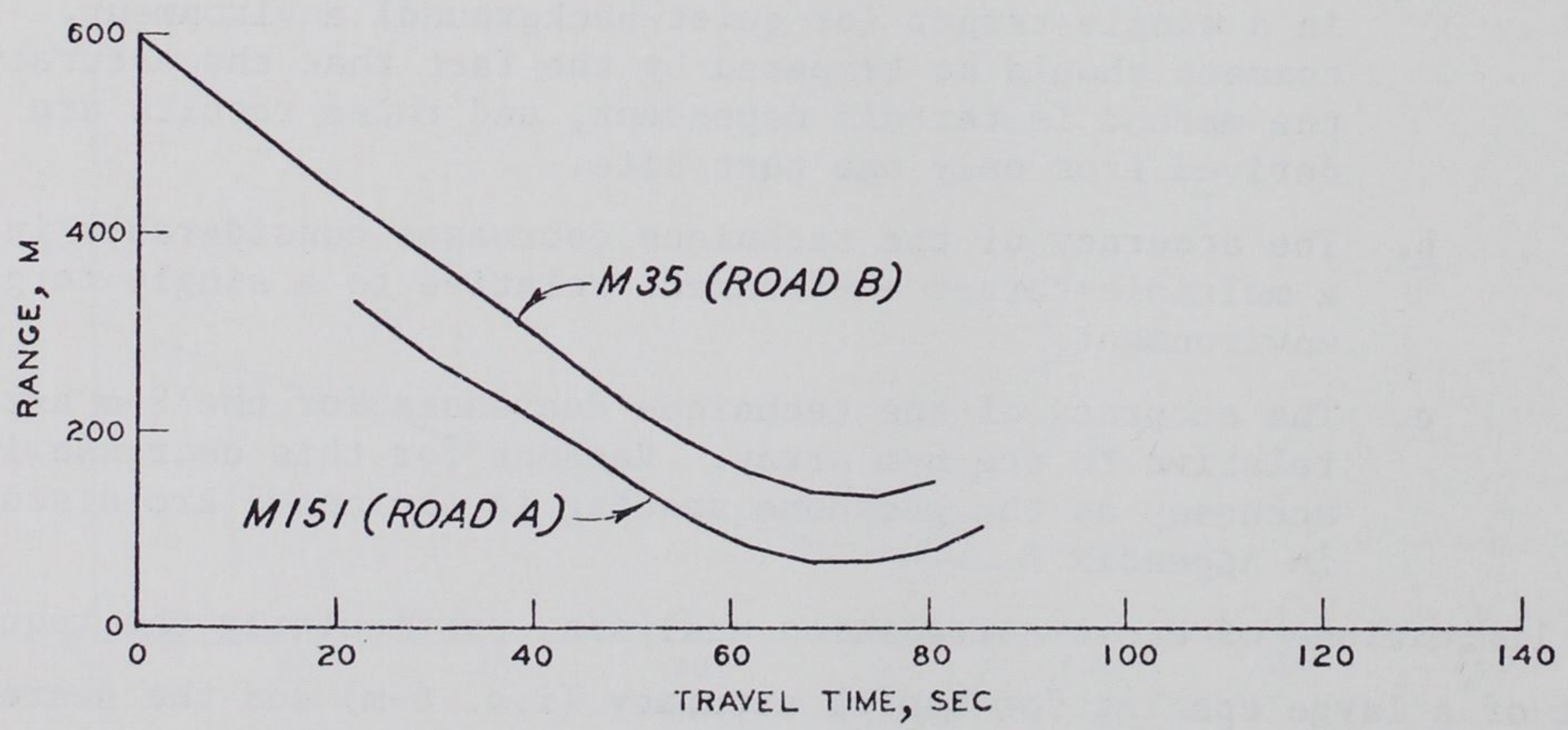

b. MEASURED RANGE FROM SENSOR ARRAY TO TARGET

Figure 13. Position information for multiple-target test involving M35 truck and Ml5l jeep (velocity $=24 \mathrm{~km} / \mathrm{hr}$, geophone spacing $=6 \mathrm{~m}$ ) using geophones 1,2 , and 3 (Figure 7) 
in accuracy in a multiple-target environment, suggest that alternative techniques of signature analysis should be investigated. Coherentsignature analysis was investigated in order to address the shortcomings of the simpler cross-correlation analysis (paragraph 17).

Results of coherent-signature analysis

33. The coherent-signature analysis was applied to data selected from the Vicksburg and Fort Carson tests as listed below. Test numbers correspond with those in Table Cl.

\begin{tabular}{|c|c|c|c|c|c|c|c|}
\hline $\begin{array}{l}\text { Test } \\
\text { No. } \\
\end{array}$ & Site & Vehicle (s) & $\begin{array}{l}\text { Velocity } \\
\mathrm{km} / \mathrm{hr} \\
\end{array}$ & Range, $\mathrm{m}$ & $\begin{array}{c}\text { Road } \\
\text { or } \\
\text { Path } \\
\end{array}$ & $\begin{array}{l}\text { Array } \\
\text { Spacing } \\
\mathrm{m} \\
\end{array}$ & $\begin{array}{c}\text { Geophone } \\
\text { Type } \\
\end{array}$ \\
\hline 1 & Vicksburg & M113 & 32 & 450 & B & 2 & $\mathrm{HS}-10$ \\
\hline 5 & Vicksburg & M35 & 16 & 185 & A & 2 & $\mathrm{HS}-10$ \\
\hline 9 & Ft. Carson & M728 & 16 & 280 & A & 2 & $L-4$ \\
\hline 7 & Vicksburg & $\begin{array}{l}\text { M35 } \\
\text { M151 }\end{array}$ & $\begin{array}{l}32 \\
24\end{array}$ & $\begin{array}{l}190 \\
100\end{array}$ & $\begin{array}{l}\text { B } \\
\text { A }\end{array}$ & 2 & HS -10 \\
\hline
\end{tabular}

These data were selected because they include signatures from wheeled (M151, M35), light tracked (M113), and heavy tracked (M728) vehicles; they include representative ranges and velocities and data from geologically different test sites; and two geophone types (i.e. vertical-sensing Scientific HS-10 and triaxial-sensing Mark Product L-4) are represented. (Only the vertical sensing component of the triaxial geophones was used in the analysis.) The 2-m spacing between geophones in the array was used in all tests instead of the $6-\mathrm{m}$ spacing because the smaller array is less susceptible to the ambiguities in the computed phase shift (paragraph 21), particularly at the higher frequencies. As in the cross-correlation analysis, the data were digitized at $1500 \mathrm{samples} / \mathrm{sec}$, allowing a frequency analysis up to $750 \mathrm{~Hz}$. The results of the data analysis are discussed below.

34. Test 1. Test 1 was conducted at the Vicksburg site with an M113 moving at $32 \mathrm{~km} / \mathrm{hr}$ on road B at a range of $450 \mathrm{~m}$. The array consisted of HS-10 geophones spaced $2 \mathrm{~m}$ apart. Calculated directional angles for the M113 at a measured directional angle of $68.5 \mathrm{deg}$ are presented in Table 4. The corresponding frequencies and coherence 
estimates are also given in the table. For low frequencies (less than $10 \mathrm{~Hz}$ ) the calculated directional angles are consistently too sma11 in spite of the high coherence values. This discrepancy in the calculated directional angles was also typical of the results of analysis of the remaining tests and could be due to different phase response characteristics of the geophones at the low frequencies. Above $10 \mathrm{~Hz}$, the accuracy of the calculated directional angles is closely related to the coherence value. The calculated angles are consistently accurate for coherence values above 0.85 , although some calculated angles are quite accurate (i.e. within $5 \mathrm{deg}$ ) for low coherence values, such as the angle at $114.37 \mathrm{~Hz}$. The average of the calculated directional angles having coherence values greater than 0.85 and frequency above $10 \mathrm{~Hz}$ is $69.5 \mathrm{deg}$, which compares very favorably with the measured value of $68.5 \mathrm{deg}$. A polar plot showing the calculated directional angles for each frequency greater than $10 \mathrm{~Hz}$ and having coherence estimates greater than 0.85 is presented in Figure 14.

35. Test 5. Test 5 was conducted at the Vicksburg test site with an M35 moving at $16 \mathrm{~km} / \mathrm{hr}$ on road $\mathrm{A}$ at a range of $185 \mathrm{~m}$. The array consisted of HS-10 geophones spaced $2 \mathrm{~m}$ apart. Calculated directional angles for the M35 at a measured directional angle of $248 \mathrm{deg}$ are presented in Table 5 for those frequencies greater than $10 \mathrm{~Hz}$ and having associated coherence estimates greater than 0.85 . A polar plot of the calculated angles appearing in Table 5 is presented in Figure 15.

36. Test 9. Test 9 was conducted at the Fort Carson site with an M728 moving at $16 \mathrm{~km} / \mathrm{hr}$ on path A (cross-country) at a range of $280 \mathrm{~m}$. The array consisted of Mark Product L-4 geophones spaced $2 \mathrm{~m}$ apart. Calculated directional angles for the M728 at a measured directional angle of 311 deg are presented in Table 6 for those frequencies greater than $10 \mathrm{~Hz}$ and having associated coherence estimates greater than 0.85 . A polar plot of the calculated directional angles appearing in Table 6 is presented in Figure 16.

37. Test 7 . Test 7 was conducted at the Vicksburg test site with two vehicles, an M35 and an M151, moving at 32 and $24 \mathrm{~km} / \mathrm{hr}$ on roads B and $\mathrm{A}$, respectively. The array consisted of HS-10 geophones spaced $2 \mathrm{~m}$ 


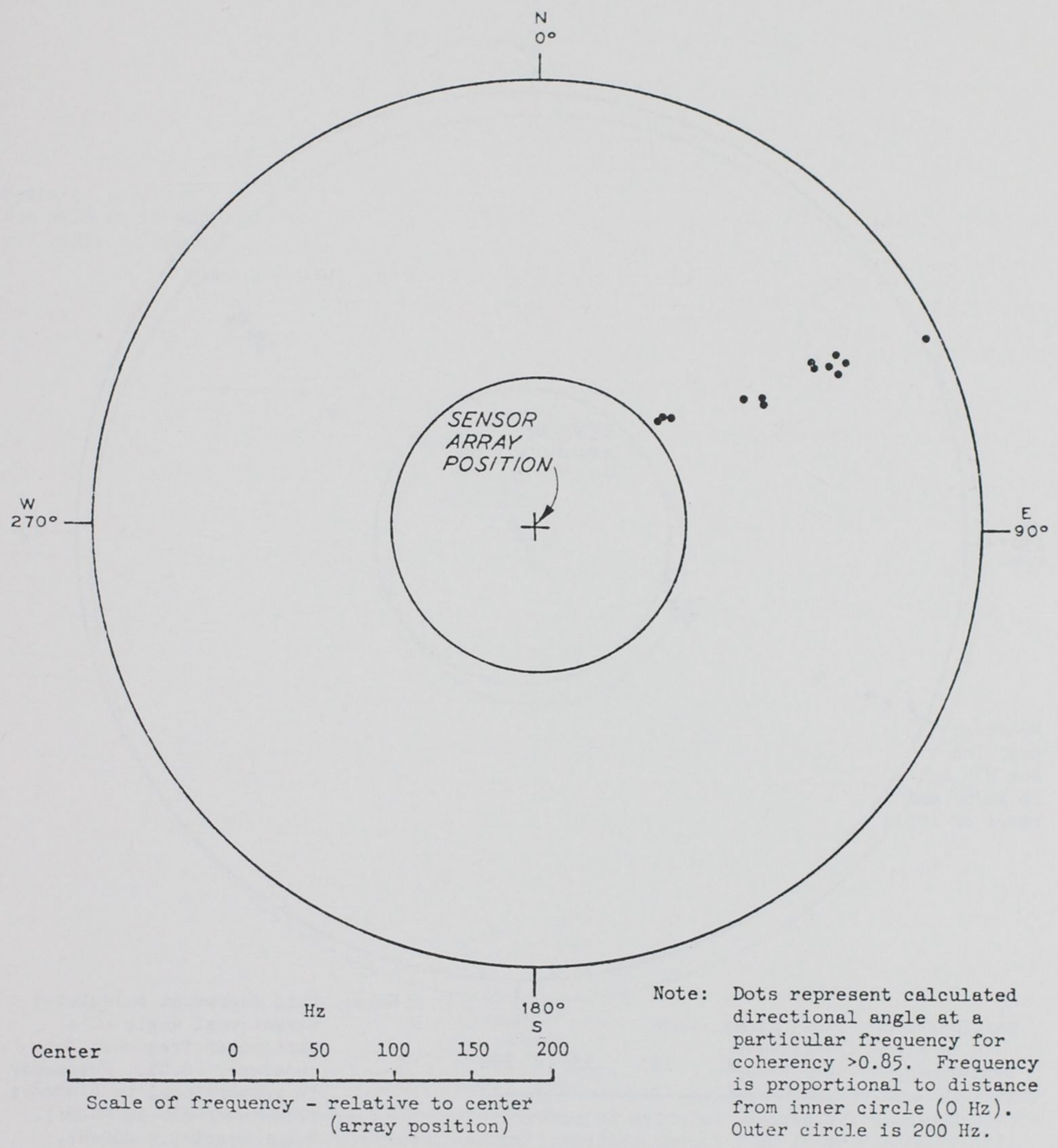

Figure 14. Polar plot of direction to an M113 at Vicksburg test site 


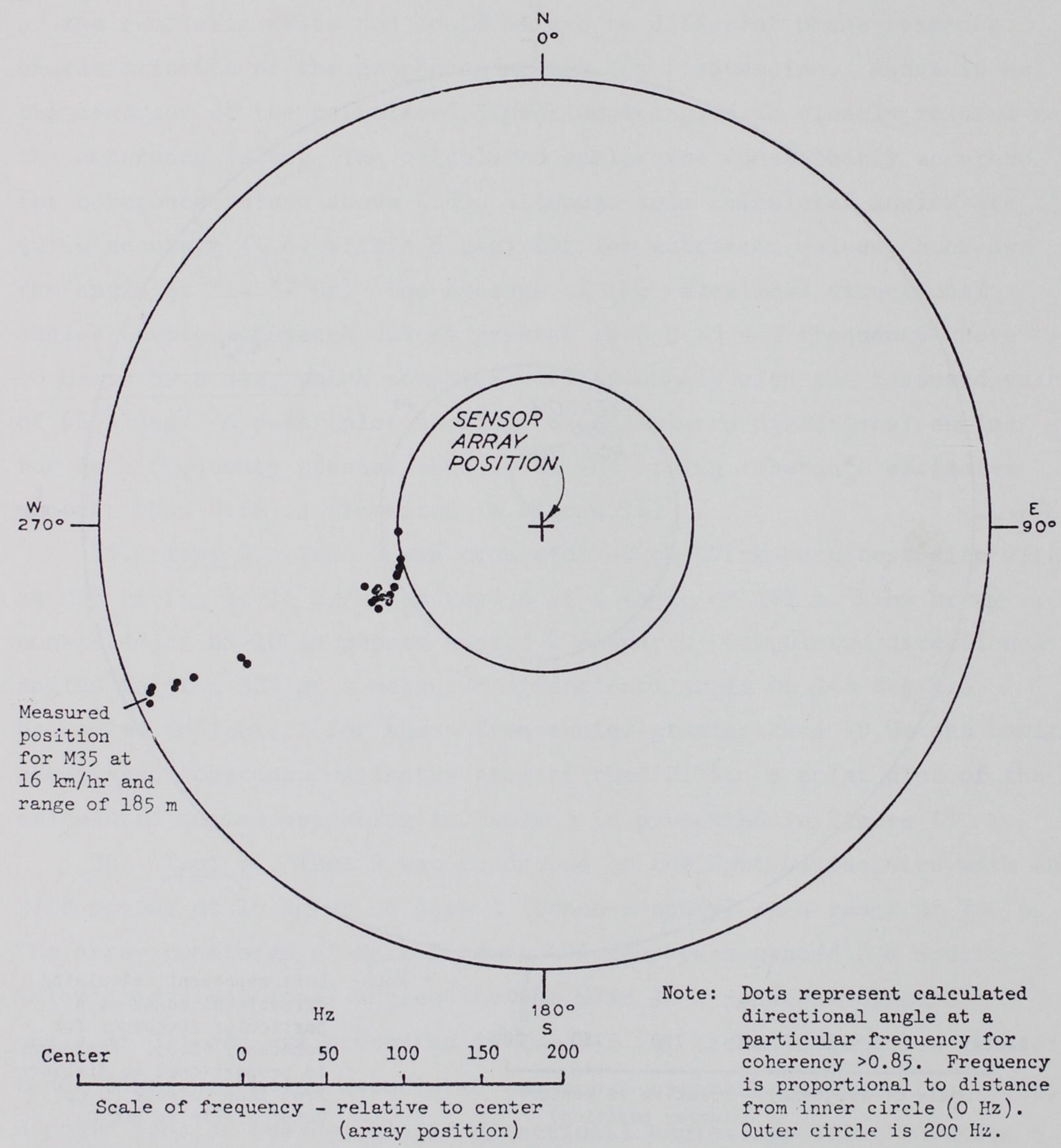

Figure 15. Polar plot of direction to an M35 at Vicksburg test site 


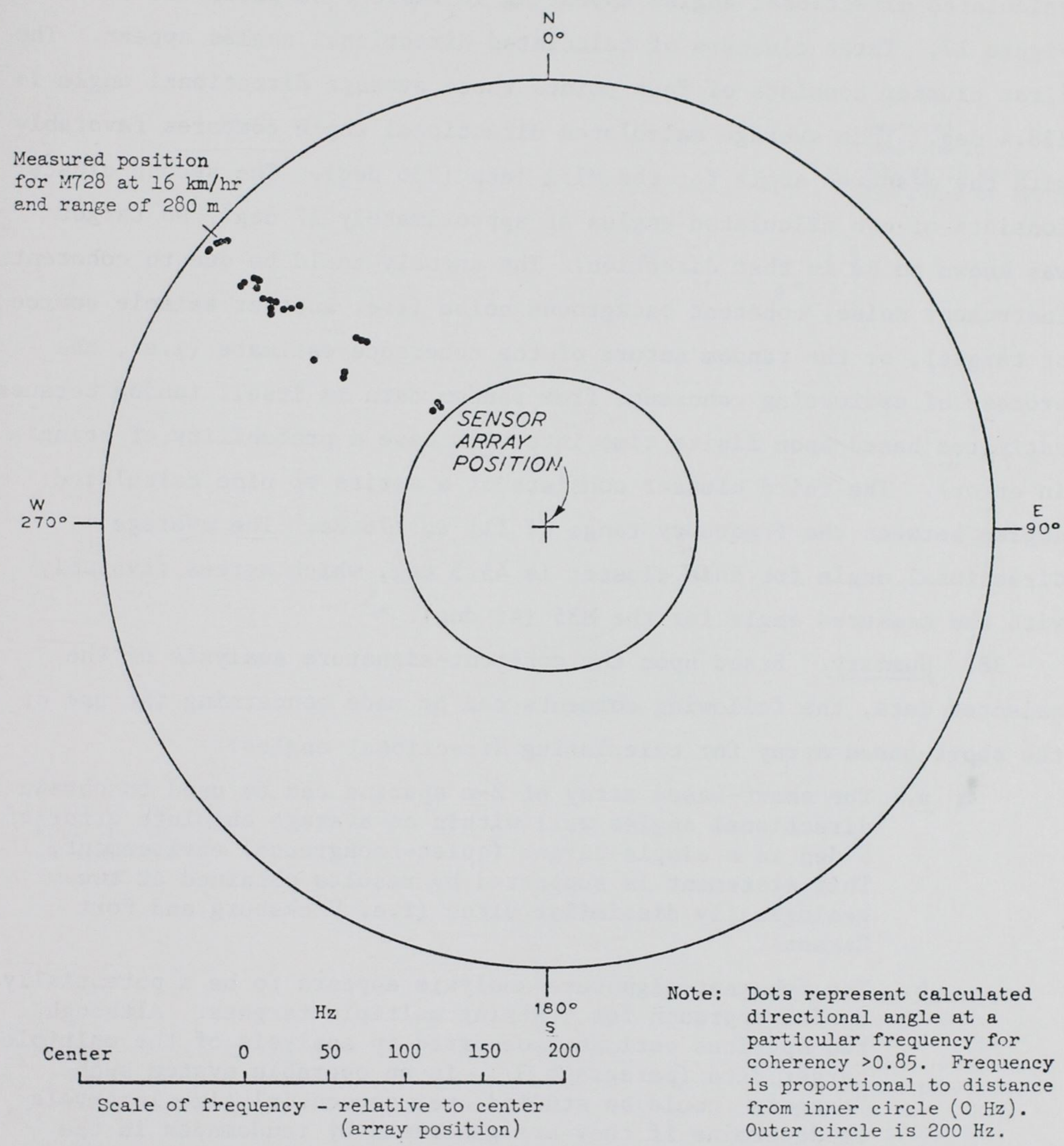

Figure 16. Polar plot of direction to M728 at Fort Carson test site 
apart. Calculated directional angles for location of the M35 at a measured angle of $45 \mathrm{deg}$ and the M151 at a measured angle of 236 deg are presented in Table 7 for those frequencies greater than $10 \mathrm{~Hz}$ and having an associated coherence estimate greater than 0.85. A polar plot of the calculated directional angles appearing in Table 7 is presented in Figure 17. Three clusters of calculated directional angles appear. The first cluster consists of four points whose average directional angle is $238.4 \mathrm{deg}$. This average calculated directional angle compares favorably with the measured angle for the M151 jeep (236 deg). The second cluster consists of two calculated angles at approximately $17 \mathrm{deg}$. No target was known to be in that direction. The anomaly could be due to coherent instrument noise, coherent background noise (i.e. another seismic source or target), or the random nature of the coherence estimate (i.e., the process of estimating coherence from random data is itself random because estimates based upon finite time intervals have a probability of being in error). The third cluster consists of a series of nine calculated angles between the frequency range of 115 to $176 \mathrm{~Hz}$. The average directional angle for this cluster is $45.5 \mathrm{deg}$, which agrees favorably with the measured angle for the M35 (45 deg).

38. Summary. Based upon the coherent-signature analysis of the selected data, the following comments can be made concerning the use of the short-based array for calculating directional angles:

a. The short-based array of $2-m$ spacing can be used to obtain directional angles well within an average absolute error of 5 deg in a single-target (quiet-background) environment. This statement is supported by results obtained at two geologically dissimilar sites (i.e. Vicksburg and Fort Carson).

b. The coherent-signature analysis appears to be a potentially useful approach for tracking multiple targets. Although two spurious estimates occurred in analysis of the multipletarget data (paragraph 37), in an operable system such "targets" could be studied over sequential time intervals to determine if they are generated by randomness in the directional angles, by coherent background noise in the array environment, or by a "target" having characteristics (i.e. velocity, frequency, range, etc.) of the targets of interest. 
c. Seismic signatures produced by wheeled and tracked vehicles contain sufficient energy between 0 and $200 \mathrm{~Hz}$ to permit computation of directional angles at ranges at least as large as $450 \mathrm{~m}$ (Test 1, paragraph 34).

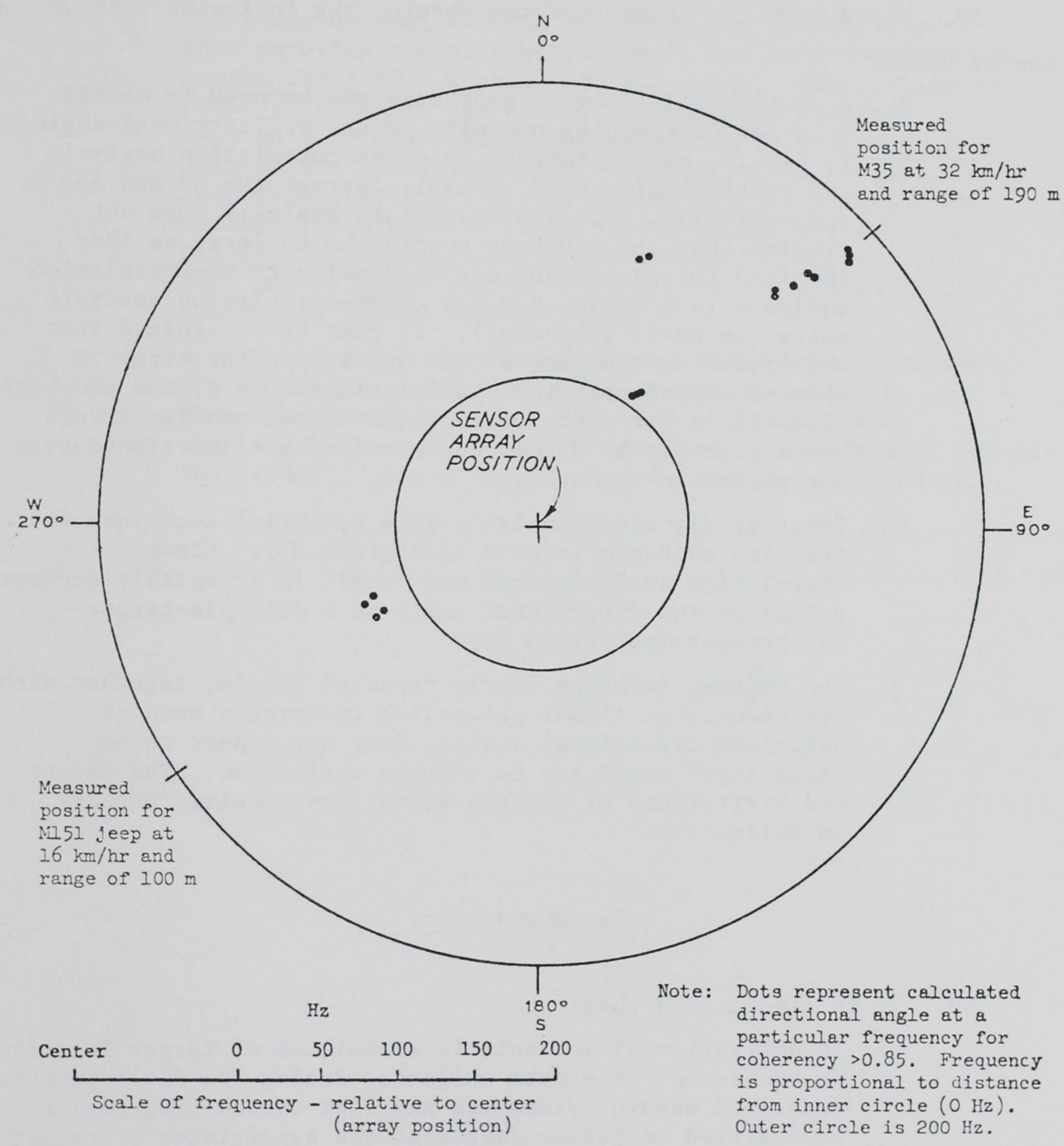

Figure 17. Polar plot of directions to multiple targets consisting of an M35 and an M151 


\section{PART IV: CONCLUSIONS AND RECOMMENDATIONS}

\section{Conclusions}

39. Based upon the study reported herein, the following conclusions can be drawn:

a. A short-based array of geophones can be used to obtain data for determining usefully accurate directional angles to single, moving targets by cross-correlation analysis and coherent-signature analysis (paragraphs 32 and 38, respectively). Coherent-signature analysis does not require that the geophone spacing be as large as that required for equivalent accuracy using cross-correlation analysis ( $2 \mathrm{~m}$ versus $6 \mathrm{~m}$ for cross-correlation analysis using the HS-10 geophones). It must be recognized that the limits on accuracy as the spacing in the array is changed depend upon the spatial dimensions of the geophones (i.e. $18 \mathrm{~cm}$ for a HS-10) and, therefore, smaller arrays could probably be developed, provided smaller transducers are placed in the array.

b. Coherent-signature analysis is a potential technique for tracking multiple targets (paragraph 37). Crosscorrelation analysis does not result in acceptably accurate values of the directional angle in a multiple-target environment (paragraph 31).

c. The seismic location system reported herein, together with its associated signal-processing techniques used to calculate directional angles, does not appear to be exceedingly sensitive to terrain variations. The extent and limitations of terrain variations require further investigation.

\section{$\underline{\text { Recommendations }}$}

40. It is recommended that:

a. Investigation of the seismic techniques of target location be continued. The data collected during the field program described herein (Vicksburg and Fort Carson programs) should be analyzed to better determine the sensitivity of target location methods to terrain variation. Furthermore, as the data on hand are not extensive, additional data should be collected at geologically dissimilar sites, and these data should be analyzed as described above. 
b. Instrumentation response characteristics, including those of small seismic transducers, be investigated to determine the state-of-the-art capability of constructing a shortbased array of minimum spatial dimensions. The sensitivity of the directional algorithm to changes in design criteria could be evaluated as discussed in Appendix A.

c. The study be continued to determine the feasibility of incorporating target classification and location into the capabilities of a single geophone array. The utility of combined acoustic and seismic sensors for ranging (Appendix B) and classification should be included; in particular, the feasibility of applying the ranging technique (paragraph 7b) to firing artillery should be further investigated. Alternative techniques of signal processing, in addition to those reported herein, that make use of phase shift and amplitude properties of the signatures should be considered.

d. A study be initiated to define the performance envelope for competing or supplementing techniques, such as acoustic techniques, as a function of terrain and vegetation cover in order to compare (or supplement) seismic and acoustic approaches to target location efficiently. 
Table 1

Position Information for M113 APC Using

2- and 6-m Arrays

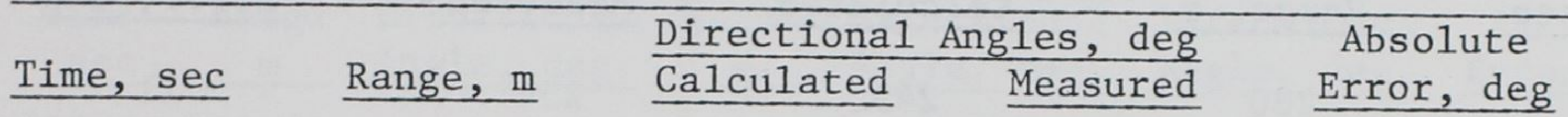

16.6

410

2-m Array

22.5

365

28.5

315

34.4

270

40.3

215

45.9

190

51.7

155

57.5

130

10

15

11

17

19

19

22

25

29

36

44

63.5

130

70.0

145

59

52

74

5

6

0

2

45

20

36

6

42

6

72

29

7

2

Avg 8.3

6-m Array

$0 \quad 600$

10

12

2

5.23

510

12

13

1

16.6

410

14

15

1

22.5

365

14

17

3

28.5

315

17

19

2

34.4

270

31

22

9

40.3

215

45.9

190

51.7

155

57.5

130

39

25

14

63.5

130

34

29

5

41

36

5

48

44

4

70.0

145

58

52

6

73

74

1

Avg 4.4 
Table 2

Position Information for M35 Using 6-m Array

\begin{tabular}{|c|c|c|c|c|}
\hline Time, sec & Range, m & $\begin{array}{l}\text { Directiona } \\
\text { Calculated }\end{array}$ & $\frac{\text { gle, deg }}{\text { Measured }}$ & $\begin{array}{c}\text { Absolute } \\
\text { Error, deg }\end{array}$ \\
\hline 15.9 & 280 & 245 & 256 & 11 \\
\hline 29.5 & 230 & 256 & 254 & 2 \\
\hline 44.4 & 180 & 243 & 250 & 7 \\
\hline 60.2 & 135 & 241 & 242 & 1 \\
\hline 74.4 & 90 & 231 & 228 & 3 \\
\hline 90.1 & 70 & 200 & 197 & 3 \\
\hline 105.3 & 105 & 159 & 154 & 5 \\
\hline 120.4 & 145 & 134 & 128 & 6 \\
\hline
\end{tabular}


Table 3

Position Information for Multiple Targets Using 6-m Array

\begin{tabular}{|c|c|c|c|c|c|c|}
\hline \multirow[b]{2}{*}{$\begin{array}{r}\text { Time } \\
\text { sec } \\
\end{array}$} & \multicolumn{2}{|c|}{ M35 Location } & \multicolumn{2}{|c|}{ M151 Location } & \multirow{2}{*}{$\begin{array}{l}\text { Calculated } \\
\text { Directional } \\
\text { Angle, deg } \\
\end{array}$} & \multirow[b]{2}{*}{$\begin{array}{l}\text { Absolute } \\
\text { Error,* deg }\end{array}$} \\
\hline & $\begin{array}{c}\text { Range } \\
\mathrm{m} \\
\end{array}$ & $\begin{array}{l}\text { Directional } \\
\text { Angle, deg } \\
\end{array}$ & $\begin{array}{c}\text { Range } \\
\mathrm{m}\end{array}$ & $\begin{array}{l}\text { Directional } \\
\text { Angle, deg } \\
\end{array}$ & & \\
\hline 18.3 & 410 & 15 & 210 & 253 & 6 & 9 \\
\hline 25.3 & 365 & 17 & 165 & 248 & 59 & 42 \\
\hline 31.3 & 315 & 19 & 131 & 241 & 36 & 17 \\
\hline 38.1 & 270 & 22 & 93 & 230 & 55 & 33 \\
\hline 43.5 & 215 & 25 & 78 & 212 & 32 & 7 \\
\hline 48.9 & 190 & 29 & 80 & 185 & 44 & 15 \\
\hline 55.3 & 155 & 36 & 107 & 150 & 60 & 24 \\
\hline 62.4 & 130 & 44 & 144 & 138 & 56 & 12 \\
\hline 68.3 & 130 & 52 & ** & ** & 70 & 18 \\
\hline 74.2 & 145 & 74 & ** & ** & 58 & $\begin{array}{c}26 \\
\text { Avg } 20.3\end{array}$ \\
\hline
\end{tabular}

* Error is computed using M35 directional angles because crosscorrelation analysis technique results in computed angles that "attempt" to track only the M35.

** M151 completed passage on road A (Figure 8) at $62.7 \mathrm{sec}$ into test run. Vehicle continued moving for several seconds after completion time. 
Table 4

Directional Angles Calculated for M113 APC at

Measured Angle of $68.5 \mathrm{deg}$, Using 2-m Array

\begin{tabular}{|c|c|c|}
\hline Frequency, $\mathrm{Hz}$ & Coherence & $\begin{array}{c}\text { Calculated Directiona1 } \\
\text { Angle, deg } \\
\end{array}$ \\
\hline 2.93 & 0.97 & 63.2 \\
\hline 4.39 & 0.99 & 51.4 \\
\hline 5.86 & 0.99 & 52.2 \\
\hline 7.33 & 0.99 & 52.8 \\
\hline 8.79 & 0.99 & 53.3 \\
\hline 10.26 & 0.99 & 63.9 \\
\hline 11.73 & 0.97 & 70.0 \\
\hline 13.20 & 0.95 & 74.5 \\
\hline 14.66 & 0.92 & 69.6 \\
\hline 16.13 & 0.82 & 60.2 \\
\hline 17.59 & 0.68 & 52.6 \\
\hline 19.06 & 0.73 & 64.8 \\
\hline 20.52 & 0.68 & 49.9 \\
\hline 21.99 & 0.61 & 25.4 \\
\hline 23.46 & 0.61 & 22.2 \\
\hline 24.92 & 0.62 & 134.5 \\
\hline 26.39 & 0.60 & 73.5 \\
\hline - & - & - \\
\hline - & - & - \\
\hline - & - & - \\
\hline 68.92 & 0.46 & 86.2 \\
\hline 70.38 & 0.51 & 252.6 \\
\hline 71.85 & 0.21 & 275.1 \\
\hline 73.31 & 0.40 & 5.8 \\
\hline 74.78 & 0.43 & 11.4 \\
\hline 76.25 & 0.81 & 66.9 \\
\hline 77.71 & 0.91 & 73.5 \\
\hline
\end{tabular}


Table 4 (Concluded)

Frequency, $\mathrm{Hz}$

107.04

108.51

109.97

111.14

112.91

114.37

115.84

\section{Coherence}

0.50

0.57

0.79

0.90

0.90

0.65

0.46

$\bullet$
Calculated Directional Angle, deg

289.2

290.2

295.3

66.3

68.3

72.9

78.0 
Table 5

Directional Angles Calculated for M35 at

Measured Angle of $248 \mathrm{deg}$, Using 2-m Array

\begin{tabular}{|c|c|c|}
\hline Frequency, $\mathrm{Hz}$ & Coherence & $\begin{array}{c}\text { Calculated Directional } \\
\text { Angle, deg }\end{array}$ \\
\hline 10.26 & 0.98 & 249.6 \\
\hline 11.73 & 0.99 & 249.9 \\
\hline 13.19 & 0.98 & 250.2 \\
\hline 14.66 & 0.98 & 247.5 \\
\hline 16.13 & 0.99 & 247.2 \\
\hline 19.06 & 0.97 & 246.5 \\
\hline 20.53 & 0.91 & 245.3 \\
\hline 21.99 & 0.92 & 248.6 \\
\hline 23.46 & 0.95 & 250.7 \\
\hline 24.92 & 0.96 & 250.7 \\
\hline 26.39 & 0.97 & 248.7 \\
\hline 27.86 & 0.97 & 246.1 \\
\hline 29.32 & 0.97 & 245.7 \\
\hline - & - & - \\
\hline - & - & - \\
\hline - & - & - \\
\hline 158.36 & 0.86 & 247.6 \\
\hline 173.02 & 0.91 & 247.4 \\
\hline 174.49 & 0.93 & 247.3 \\
\hline 178.89 & 0.91 & 248.4 \\
\hline 180.36 & 0.92 & 247.5 \\
\hline 181.82 & 0.89 & 245.5 \\
\hline
\end{tabular}


Table 6

Directional Angles Calculated for M728 at

Measured Angle of $311 \mathrm{deg}$, Using 2-m Array

\begin{tabular}{|c|c|c|}
\hline Frequency, $\mathrm{Hz}$ & Coherence & $\begin{array}{c}\text { Calculated } \\
\text { Directional Angle, deg }\end{array}$ \\
\hline 11.72 & 0.99 & 315.8 \\
\hline 13.67 & 0.98 & 313.8 \\
\hline 15.62 & 0.95 & 316.2 \\
\hline 76.17 & 0.89 & 305.4 \\
\hline 80.01 & 0.86 & 314.2 \\
\hline 82.03 & 0.91 & 314.3 \\
\hline 83.98 & 0.93 & 314.1 \\
\hline 85.94 & 0.92 & 313.7 \\
\hline 97.66 & 0.85 & 304.3 \\
\hline 128.91 & 0.85 & 310.8 \\
\hline 130.86 & 0.88 & 310.4 \\
\hline 132.81 & 0.90 & 309.5 \\
\hline 134.77 & 0.86 & 308.4 \\
\hline 140.63 & 0.87 & 306.7 \\
\hline 142.58 & 0.90 & 307.2 \\
\hline 144.53 & 0.97 & 308.5 \\
\hline 146.48 & 0.98 & 308.5 \\
\hline 148.44 & 0.98 & 308.1 \\
\hline 150.39 & 0.98 & 307.6 \\
\hline 152.34 & 0.97 & 307.3 \\
\hline 156.25 & 0.94 & 308.1 \\
\hline 158.20 & 0.88 & 308.3 \\
\hline 160.16 & 0.92 & 309.2 \\
\hline 162.11 & 0.95 & 309.6 \\
\hline 164.06 & 0.95 & 309.0 \\
\hline 166.02 & 0.95 & 308.0 \\
\hline
\end{tabular}

(Continued) 
Table 6 (Concluded)

Frequency, $\mathrm{Hz}$

167.97

193. 36

195.31

197.27

199.22
Coherence

0.87

0.92

0.95

0.92

0.92
Calculated

Directional Angle, deg

307.3

310.8

309.6

309.6

308.4 
Table 7

Directional Angles Calculated for

Multiple-Target Tests, Using 2-m Array

\begin{tabular}{ccc}
\hline Frequency, $\mathrm{Hz}$ & Coherence & $\begin{array}{c}\text { Calculated } \\
\text { Directional Angle, deg }\end{array}$ \\
\cline { 2 - 3 } 26.39 & 0.88 & 236.2 \\
27.86 & 0.88 & 241.4 \\
32.36 & 0.89 & 236.0 \\
33.72 & 0.89 & 240.0 \\
86.51 & 0.96 & 16.6 \\
87.99 & 0.95 & 18.0 \\
115.84 & 0.90 & 43.9 \\
117.30 & 0.90 & 42.9 \\
130.50 & 0.90 & 44.8 \\
131.97 & 0.92 & 44.8 \\
145.16 & 0.87 & 44.8 \\
146.63 & 0.89 & 45.8 \\
173.02 & 0.95 & 48.0 \\
174.50 & 0.97 & 47.6 \\
175.96 & 0.94 & 46.6
\end{tabular}




\section{APPENDIX A: SENSITIVITY OF THE DIRECTIONAL ALGORITHM TO TEMPORAL AND SPATIAL ERRORS}

1. The directional algorithm for using a short-based array of geophones is susceptible to several sources of error. Generally, these errors can be separated into two categories: (a) instrumentation and signal-processing errors, and (b) wave propagation errors. Errors in the first category are usually well defined and include errors arising from the spatial response of the geophones (i.e., transducers sample an area of space rather than a point). Errors in the second category are generally defined by empirical analysis. However, an analysis of propagation errors due to deviations from the plane wave approximation (i.e., the wave is more accurately described in two dimensions by a circle) can be analytically examined.

\section{Instrumentation and Signa1-Processing Errors}

2. The algorithm for using an array of geophones placed at the vertices of an equilateral triangle is given by Equation 11 of the main text as

$$
\phi=\tan ^{-1}\left[\frac{2}{\sqrt{3}}\left(\frac{{ }^{\tau} \mathrm{AB}}{\tau \mathrm{AC}}-\frac{1}{2}\right)\right]
$$

3. Assuming either that the phase response of the instrumentation is specified by a known value or that the accuracy of the signalprocessing technique for determining the phase shift between the signatures is defined, the sensitivity of the algorithm to errors in ${ }^{\tau} \mathrm{AB}$ and ${ }^{A C}$ can be determined. If $\Delta \tau$ bounds the uncertainty in the measurement of $\tau_{\mathrm{AC}}$ and ${ }^{\mathrm{AB}}$, the resulting uncertainty $\Delta \phi$ in the directional angle $\phi$ can be obtained by differentiating Equation Al. The resulting expression for $\Delta \phi$ is bounded for reasonably small values of $\Delta \tau$ by 


$$
\Delta \phi \leq \frac{\mathrm{v} \Delta \tau}{\ell} \mathrm{F}(\phi) \times(57.29 \mathrm{deg} / \mathrm{radian})
$$

with

$$
F(\phi)=(\sqrt{3}+\tan \phi) \cos \phi \quad \text { for } 0 \leq \phi \leq 60^{\circ}
$$

where $\mathrm{v}$ is the velocity of the seismic wave at a given frequency, $\ell$ is the geophone spacing, and $\Delta \phi$ is expressed in degrees. The angular dependence of the uncertainty, $F(\phi)$, is graphically presented in Figure $A 1$ and falls within the range 1.73 to 2 . The angular dependence of $F(\phi)$ in Equation $A 3$ is defined only in the region $0 \leq \phi \leq 60^{\circ}$. However, by symmetry arguments, this function can be repeated through the entire range of $\phi$.

4. As shown in Figure Al the uncertainty $\Delta \phi$, which is proportional to $F(\phi)$, is smallest when the incoming wave approaches along one of the lines formed by any two geophones in the array (i.e. along $\mathrm{AC}$ or $\mathrm{AB}$ ) and the largest when the wave approaches from an angle bisecting these lines. It should be recognized that this analysis will result in a worst-case description of the uncertainty $\Delta \phi$ because the errors in ${ }^{\tau} \mathrm{AC}$ and ${ }^{\tau} \mathrm{AB}$ are superimposed in the most destructive manner.

5. The inequality (Equation A2) can be used to obtain design parameters in the following manner. Suppose that the design criteria are that $\Delta \phi$ be less than $5 \mathrm{deg}$ and the array dimensions be less than $0.5 \mathrm{~m}$. Suppose further that the wave velocity in the operational environment is $400 \mathrm{~m} / \mathrm{sec}$ in the frequency range of interest. Since $\mathrm{F}(\phi) \leq 2$, the inequality can be used to define the accuracy requirement on the time measurements (i.e. ${ }^{\tau} \mathrm{AB}$ and ${ }^{\tau}{ }_{\mathrm{AC}}$ ) as being less than 50 microseconds $\left(50 \times 10^{-6} \mathrm{sec}\right)$. The response time of the instrumentation system could then be specified as being less than 50 microseconds for frequencies up to $400 \mathrm{~Hz}$. The cutoff frequency of $400 \mathrm{~Hz}$ is convenient because ambiguities in the calculated phase do not occur below that value (i.e., the spacing in the array is less than half the wavelength for frequencies below $400 \mathrm{~Hz}$ ). 


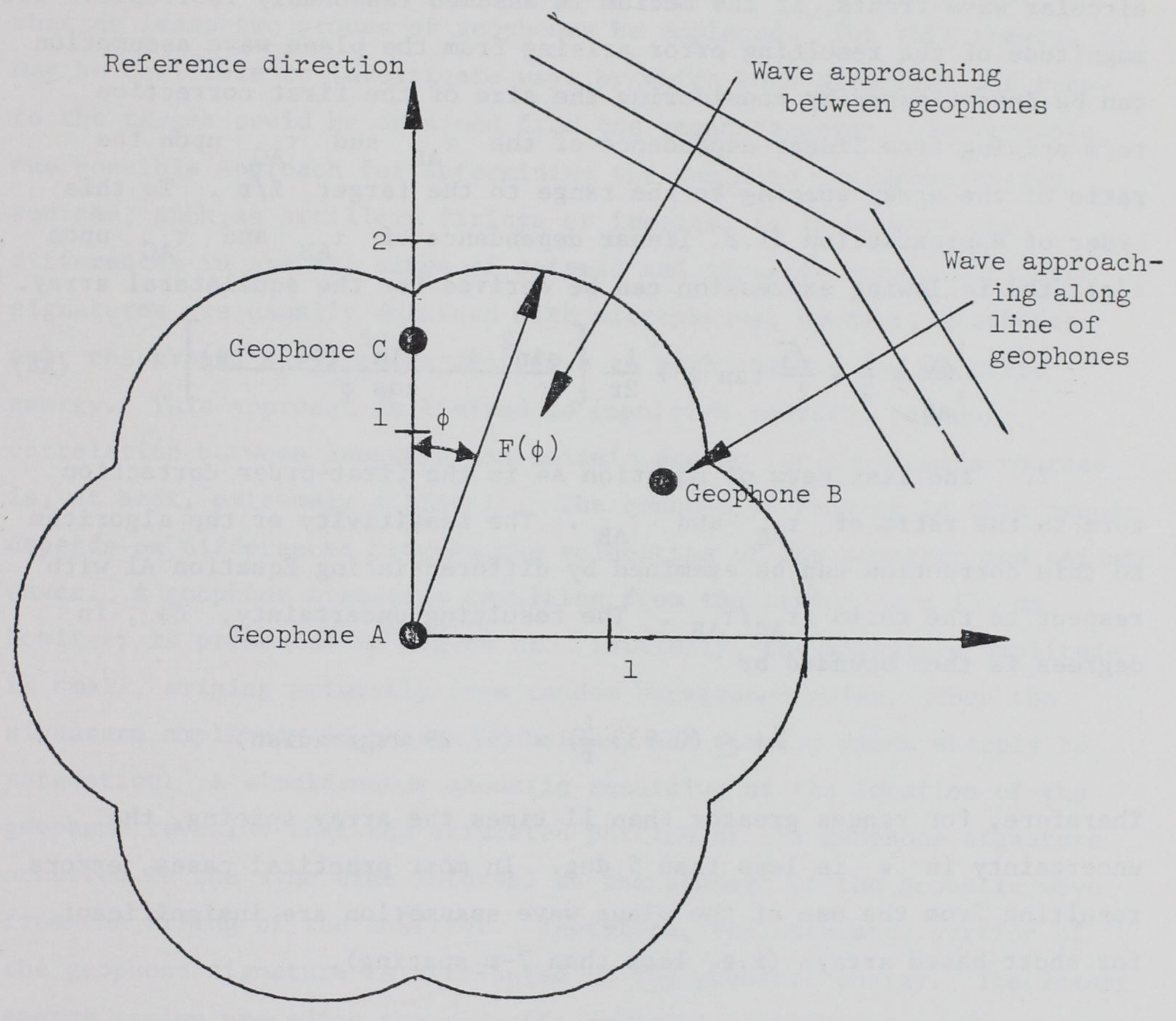

Figure Al. Angular dependence of the uncertainty in the directional measurement, $F(\phi) . F(\phi)$ is defined by Equation A3. 


\section{Errors Due to Plane Waye Assumption}

6. As described previously, the derivation of Equations 10 and 11 in the main text depends on the assumption that the approaching waves are plane waves. They are actually described, in two dimensions, by circular wave fronts, if the medium is assumed reasonably isotropic. The magnitude of the resulting error arising from the plane wave assumption can be investigated by considering the size of the first correction term arising from linear dependence of the $\tau_{A B}$ and ${ }^{\tau} A C$ upon the ratio of the array spacing to the range to the target $\ell / r$. To this order of approximation (i.e. linear dependence of ${ }^{\tau}{ }_{A B}$ and ${ }^{\tau} A C$ upon $\ell / r)$, the following expression can be derived for the equilateral array.

$$
\frac{{ }^{\tau} \mathrm{AC}}{\tau_{\mathrm{AB}}}=\frac{1}{2}+\frac{\sqrt{3}}{2} \tan \phi+\frac{\ell}{2 r}\left[\frac{\sin ^{2} \phi-\sin ^{2}\left(60^{\circ}-\phi\right)}{\cos \phi}\right]
$$

7. The last term of Equation $\mathrm{A} 4$ is the first-order correction term to the ratio of ${ }^{\tau} \mathrm{AC}$ and $\tau_{\mathrm{AB}}$. The sensitivity of the algorithm to this correction can be examined by differentiating Equation Al with respect to the ratio ${ }^{A C} / \tau_{A B}$. The resulting uncertainty, $\Delta \phi$, in degrees is then bounded by

$$
\Delta \phi \leq\left(0.933 \frac{l}{\mathrm{r}}\right) \times(57.29 \mathrm{deg} / \mathrm{radian})
$$

Therefore, for ranges greater than 11 times the array spacing, the uncertainty in $\phi$ is less than $5 \mathrm{deg}$. In most practical cases, errors resulting from the use of the plane wave assumption are insignificant for short-based arrays (i.e. less than 7-m spacing). 


\section{APPENDIX B: TARGET LOCATION USING THE DIFFERENCES IN ARRIVAL TIMES OF SEISMIC AND ACOUSTIC WAVES}

1. Reliance on triangulation techniques for target location has serious drawbacks in a battlefield environment, because they require that at least two groups of geophones be emplaced. For this reason, it may be desirable to investigate ways by which both direction and range to the target could be obtained from one geophone array. For example, one possible approach for determining the range to impulsive energy sources, such as artillery firings or impacts, is to measure the differences in arrival times of seismic and acoustic waves. Acoustic signatures are usually obtained with microphones; however, geophones near the ground surface are sensitive to both seismic and acoustic energy. This approach is limited to impulsive sources, because correlation between acoustic and seismic energy for continuous sources is, at best, extremely difficult. The concept of ranging in this manner depends on differences between the velocities of the acoustic and seismic waves. A geophone signature resulting from the firing of a $155-\mathrm{mm}$ howitzer is presented in Figure B1. Initially, the signature amplitude is small, arising primarily from random background noise. Then the signature amplitude increases, subsides, and finally rises sharply to saturation. A simultaneous acoustic recording at the location of the geophone revealed that the saturated portion of the geophone signature occurred in the same time interval as the passage of the acoustic wave from the firing of the howitzer. Therefore, the saturated portion of the geophone signature is attributed to the acoustic energy. The lesser energy region preceding the acoustic energy is attributed to the seismic energy emanating from the firing. The leading edge of this seismically active region is not well defined. However, it precedes the time of arrival of the acoustic signature by approximately $0.5 \mathrm{sec}$. A technique needs to be developed to accurately identify the arrival time of the seismic signature. Such a technique could be based upon cross correlation of the signatures received at the array. 


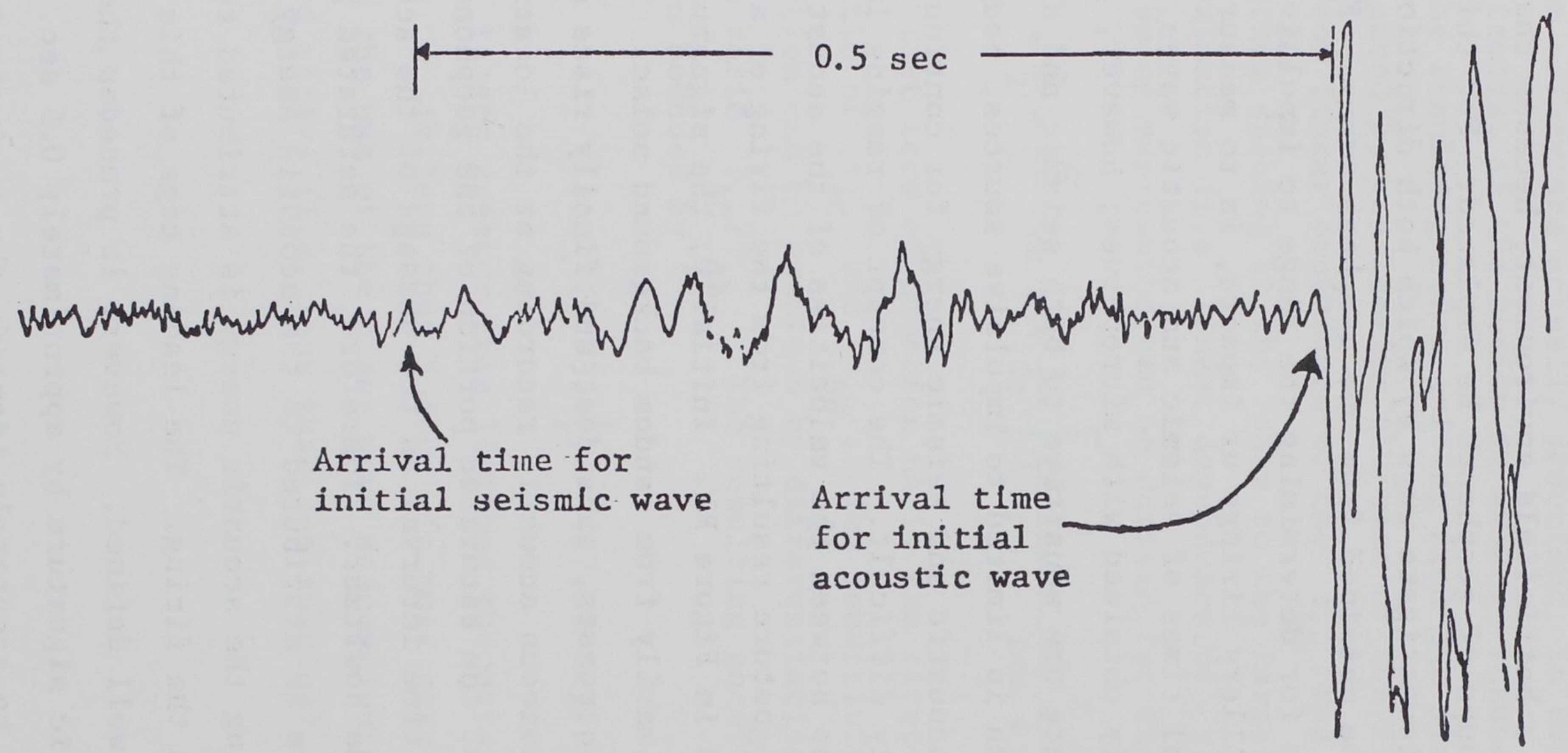

Figure BI. Difference between times of arrival of seismic and acoustic energy from a 155-mm howitzer firing. Geophone was located $1.2 \mathrm{~km}$ from the firing location 
2. A geophone array can be used to determine the direction to the target by application of the directional algorithm (Equation 11 of the main text). The velocity of the seismic wave train can be obtained from the expression

$$
v(\omega)=\frac{{ }^{{ }_{A C}} \cos \phi}{{ }^{\tau} \mathrm{AC}}
$$

which is a rearrangement of Equation 9 of the main text. The range to the target, $r$, can then be obtained from the expression

$$
r=\frac{v_{s} v_{a}}{v_{s}-v_{a}} \Delta t
$$

where

$$
\begin{aligned}
v_{s}= & \text { velocity of the seismic wave } \\
v_{a}= & \text { velocity of the acoustic wave } \\
\Delta t= & \text { difference in time between the initial receptions of the } \\
& \text { acoustic and seismic waves }
\end{aligned}
$$

The velocity of the acoustic wave in air is accurately approximated by $330 \mathrm{~m} / \mathrm{sec}$ in windless environments. For maximum accuracy the wind velocity would have to be included in the value of $v_{a}$.

3. The approach described above is potentially capable of providing a method of placing counterbattery fire on a target weapon, particularly if that weapon is in a fixed location. The procedure is hypothesized as follows:

a. The location of the enemy weapon is estimated by calculating the direction of arrival of its seismic signature, using the three-geophone array previously described. The range is obtained from the difference between the arrival times of the acoustic and seismic waves, as described in the previous paragraph (Figure B2).

b. A counterbattery weapon is then fired at the estimated location (Figure B3), and the location of the impact of that round is calculated, using the geophone array. Since the propagation paths will, in most cases, be very similar, it can be assumed that the errors in the directional angle and range will be essentially the same as the errors in the calculated location of the enemy weapon relative to its actual location. 


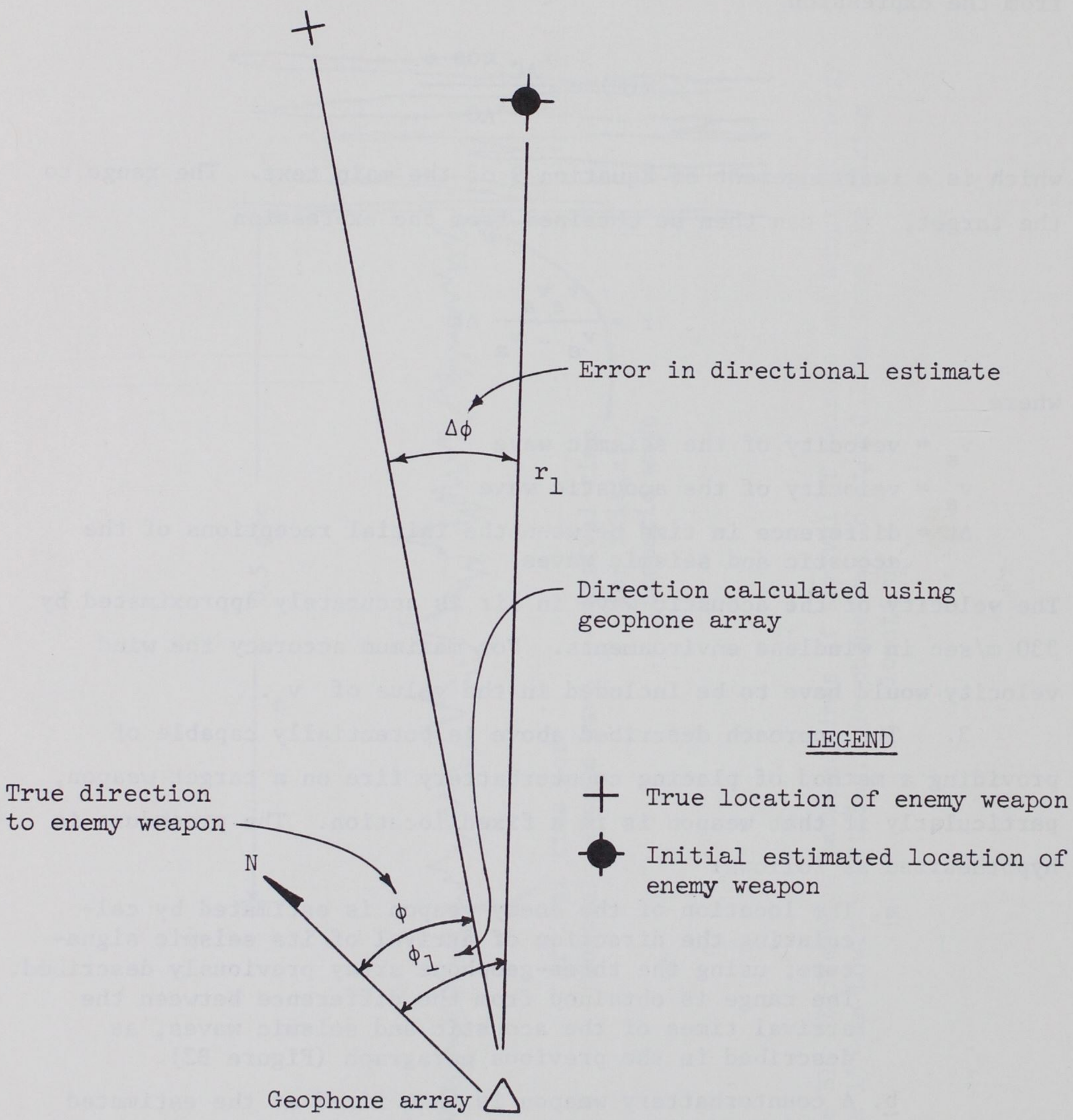

Figure B2. Initial target-location analysis 


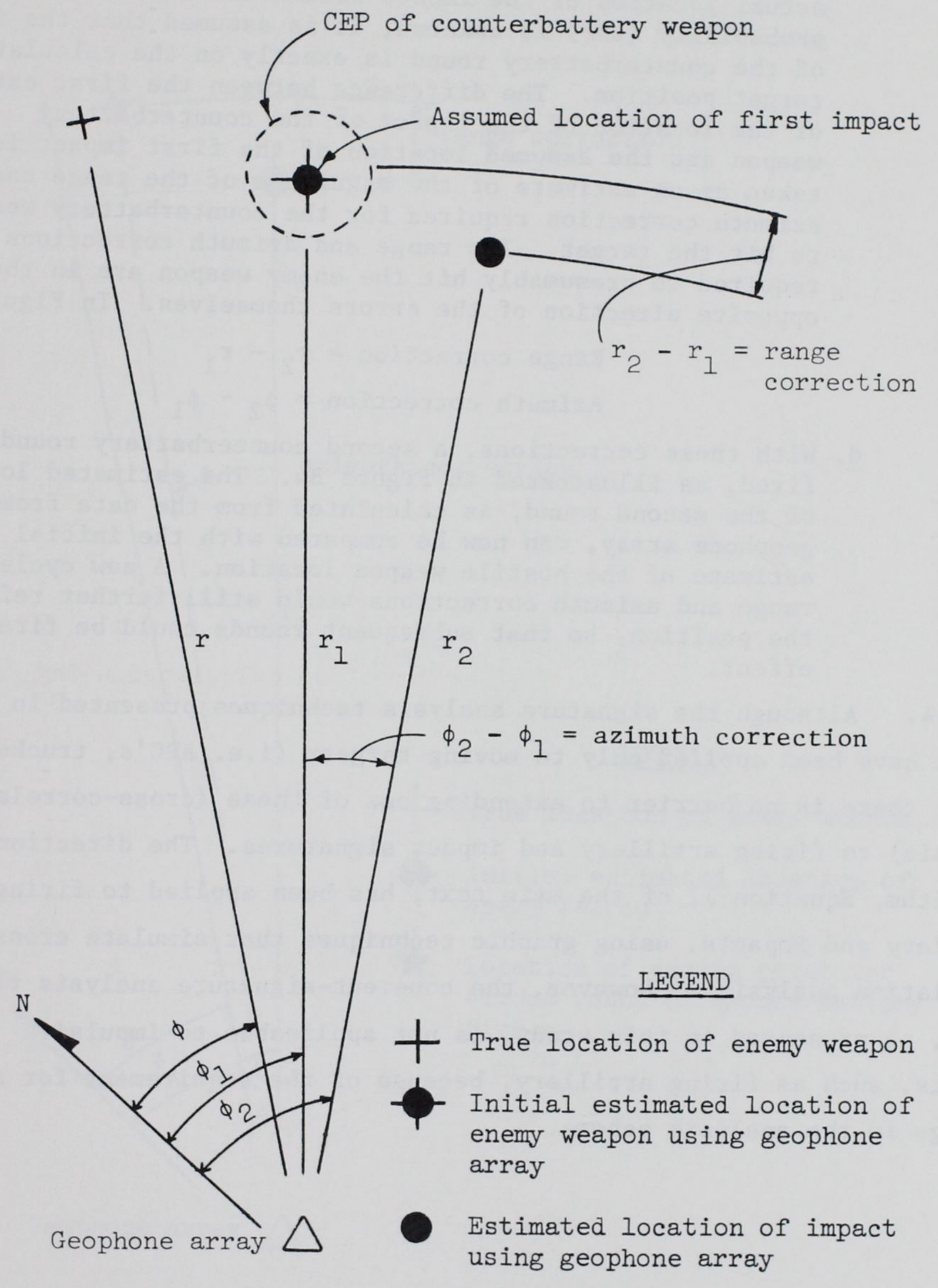

Figure B3. Calculation of position of first-round impact of counterbattery fire 
c. The location of the counterbattery impact, as calculated by the geophone array, is compared with the location of the target, as calculated in the same way. Since the actual location of the impact within the central error of probability (CEP) is unknown, it is assumed that the impact of the counterbattery round is exactly on the calculated target position. The difference between the first estimate of the location of the impact of the counterbattery weapon and the assumed location of the first impact is taken as an estimate of the magnitude of the range and azimuth correction required for the counterbattery weapon to hit the target. The range and azimuth corrections required to presumably hit the enemy weapon are in the opposite direction of the errors themselves. In Figure B3

$$
\begin{aligned}
& \text { Range correction }=r_{2}-r_{1} \\
& \text { Azimuth correction }=\phi_{2}-\phi_{1}
\end{aligned}
$$

d. With these corrections, a second counterbattery round is fired, as illustrated in Figure B4. The estimated location of the second round, as calculated from the data from the geophone array, can now be compared with the initial estimate of the hostile weapon location. A new cycle of range and azimuth corrections would still further refine the position, so that subsequent rounds could be fired for effect.

4. Although the signature analysis techniques presented in this report have been applied only to moving targets (i.e. APC's, trucks, etc.), there is no barrier to extending one of these (cross-correlation analysis) to firing artillery and impact signatures. The directional algorithm, Equation 11 of the main text, has been applied to firing artillery and impacts, using graphic techniques that simulate crosscorrelation analysis.* However, the coherent-signature analysis technique, as presented in this study, is not applicable to impulsive targets, such as firing artillery, because of the requirement for a time average in the analysis scheme.

* WESFE letter dated 27 Aug 73 to Project Manager, REMBASS, subject: "Seismic Methods for Target Location." 


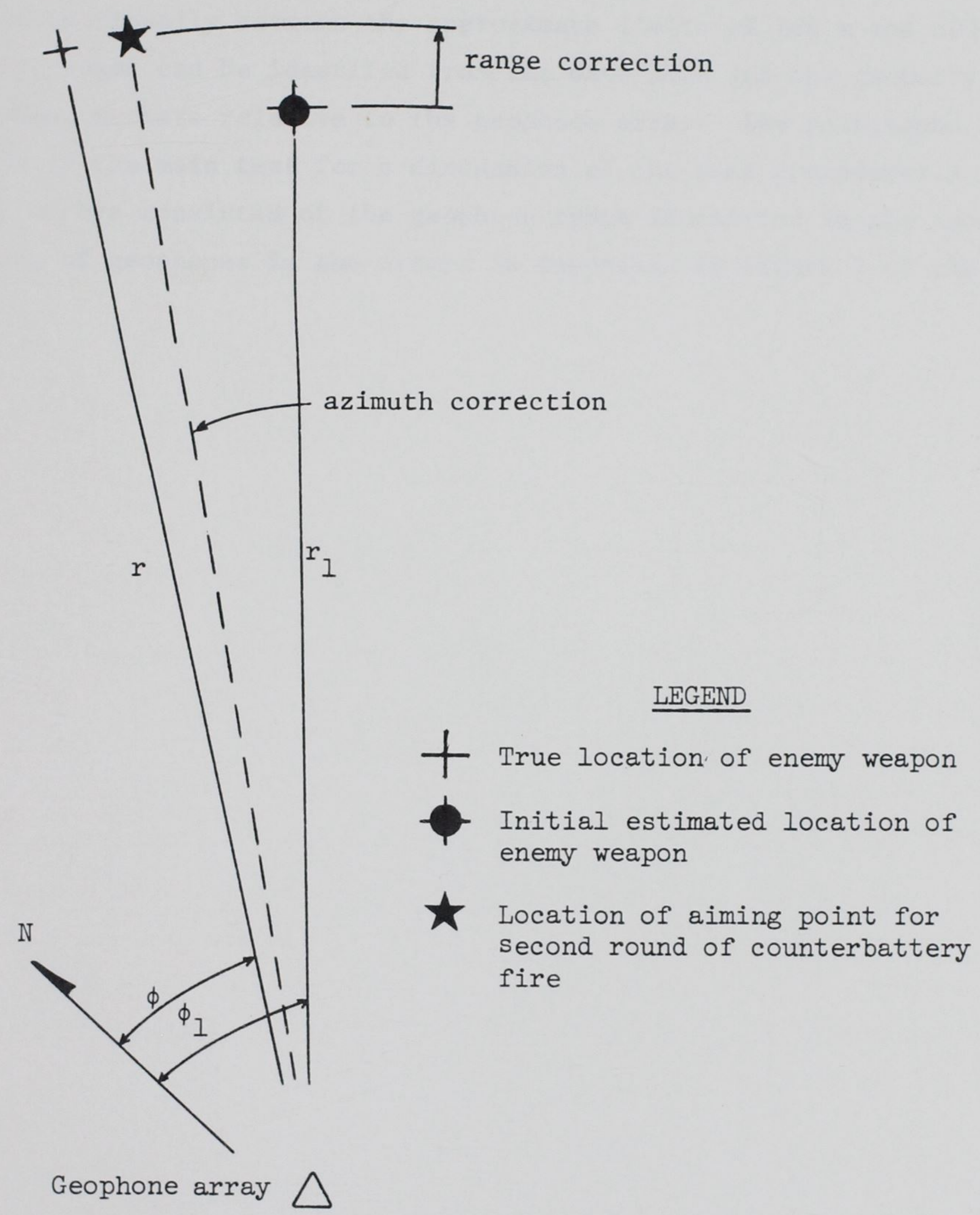

Figure B4. Calculation of range and azimuth corrections (from Figure B3) for second round of counterbattery fire 
APPENDIX C: TABULATION OF SINGLE- AND MULTIPLE-TARGET TEST CONDITIONS

1. Table Cl lists the test conditions for single- and multipletarget data collected in this program. Ranges from the geophone array varied continually between the approximate limits of $100 \mathrm{~m}$ and $600 \mathrm{~m}$. (Exact ranges can be identifed from the data logs and the geometry of the event markers relative to the geophone array. See paragraphs 23, 24, and 25 of the main text for a discussion of the test procedures.) Geophone arrays consisted of the geophone types identified in the tabulation. Spacing of geophones in the arrays is described in Figure 7 of the main text. 
Table C1

Vehicle Test Data

\begin{tabular}{|c|c|c|c|c|c|c|c|c|c|}
\hline \multirow[b]{2}{*}{ Test No. } & \multirow[b]{2}{*}{ Vehicle } & \multirow[b]{2}{*}{ Site } & \multirow[b]{2}{*}{$\begin{array}{c}\text { Geophone } \\
\text { Type } \\
\end{array}$} & \multirow[b]{2}{*}{$\begin{array}{c}\text { Spacing } \\
\mathrm{m}\end{array}$} & \multirow[b]{2}{*}{ Surface* } & \multirow[b]{2}{*}{$\begin{array}{c}\text { Velocity } \\
\mathrm{km} / \mathrm{hr}\end{array}$} & \multicolumn{3}{|c|}{ Second Target** } \\
\hline & & & & & & & Type & Surface & $\begin{array}{c}\text { Velocity } \\
\mathrm{km} / \mathrm{hr}\end{array}$ \\
\hline $\begin{array}{l}1 \\
2\end{array}$ & M113 & Vicksburg & $\mathrm{HS}-10$ & $\begin{array}{l}2 \\
6\end{array}$ & Hard & 32 & $\mathrm{NA}$ & $\mathrm{NA}$ & $\mathrm{NA}$ \\
\hline $\begin{array}{l}3 \\
4\end{array}$ & M35 & Vicksburg & HS -10 & $\begin{array}{l}2 \\
6\end{array}$ & Hard & 24 & NA & NA & NA \\
\hline $\begin{array}{l}5 \\
6\end{array}$ & & Vicksburg & HS -10 & $\begin{array}{l}2 \\
6\end{array}$ & Hard & 16 & $\mathrm{NA}$ & $\mathrm{NA}$ & $\mathrm{NA}$ \\
\hline $\begin{array}{l}7 \\
8\end{array}$ & M151 & Vicksburg & HS -10 & $\begin{array}{l}2 \\
6\end{array}$ & Hard & 24 & M35 & Hard & 32 \\
\hline $\begin{array}{r}9 \\
10 \\
11 \\
12\end{array}$ & M728 & Ft. Carson & HS -10 & $\begin{array}{l}2 \\
6 \\
2 \\
6\end{array}$ & Natural & 16 & NA & NA & NA \\
\hline $\begin{array}{l}13 \\
14 \\
15 \\
16\end{array}$ & & Ft. Carson & HS -10 & $\begin{array}{l}2 \\
6 \\
2 \\
6\end{array}$ & Natura1 & 32 & NA & NA & NA \\
\hline $\begin{array}{l}17 \\
18 \\
19 \\
20\end{array}$ & & Ft. Carson & $\mathrm{HS}-10$ & $\begin{array}{l}2 \\
6 \\
2 \\
6\end{array}$ & Hard & 24 & NA & NA & NA \\
\hline & & & & Continued) & & & & & \\
\hline
\end{tabular}

* Hard surface is a we11-used, hard packed road. Natural surface is cross-country travel.

** Second target is the associated multiple target. NA indicates that the test was a single-target test. 
Table C1 (Concluded)

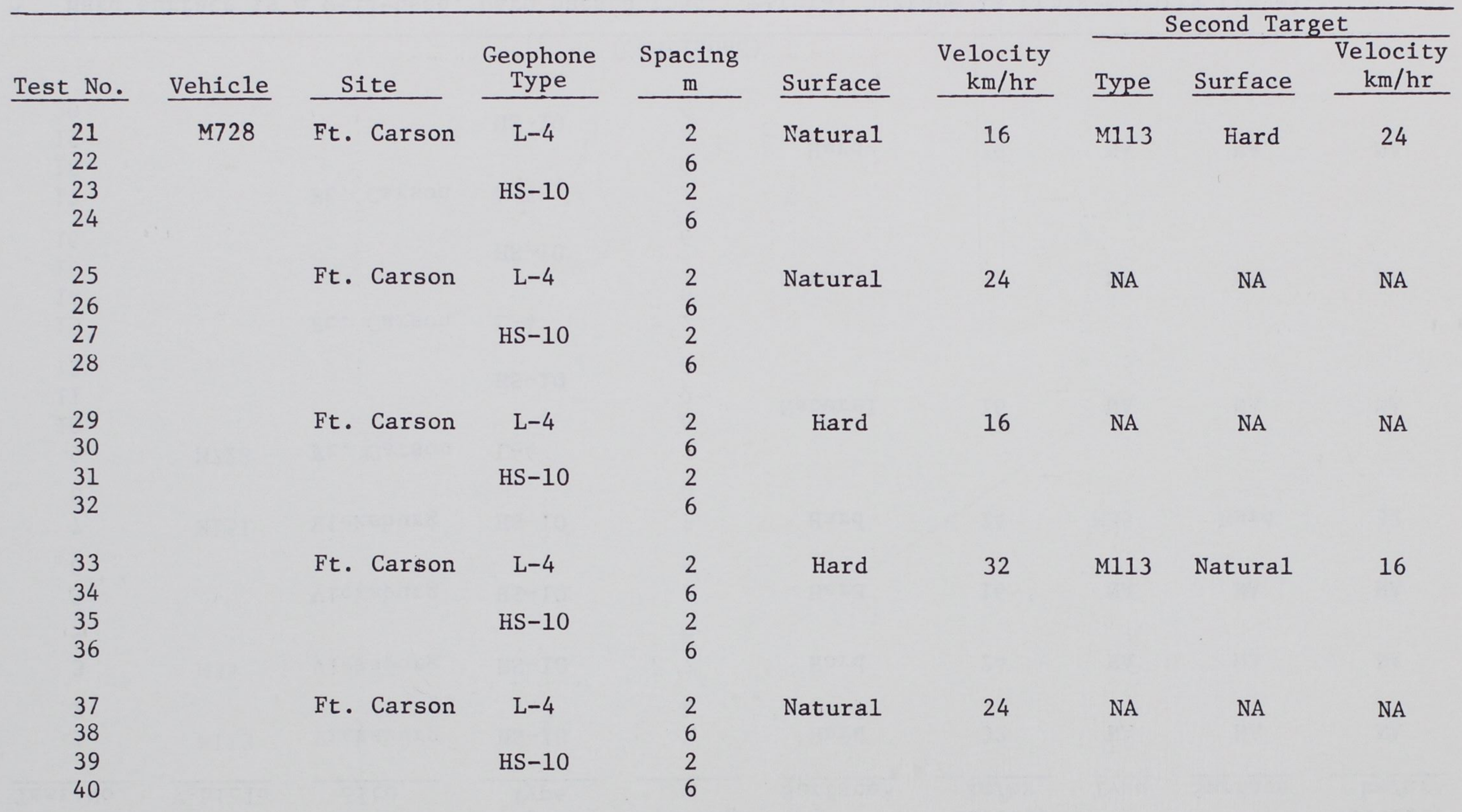

